\title{
STABLE HOMOTOPY CATEGORIES ${ }^{1,2}$
}

\author{
BY ALEX HELLER
}

Introduction. The Freudenthal suspension theorem implies that the set of homotopy classes of continuous maps from one finite complex to another is eventually invariant under iterated suspension of the complexes. In this "stable range" the set of homotopy classes is also well behaved in other ways. For example, it has in a natural way the structure of an abelian group. These stable groups of homotopy classes form the subject matter of stable homotopy theory. The most convenient way to describe them is as the colimit under successive suspensions of the set of homotopy classes. This construction, introduced by Spanier and Whitehead [19], gives interesting objects even for pairs of spaces for which the hypotheses of the Freudenthal theorem fail.

In this way the stable homotopy category-or rather categories, since the class of spaces considered may be chosen in many ways or even enlarged to encompass objects, such as spectra, more general than spaces-is defined. Stable homotopy categories have been extensively studied, being the natural loci of such phenomena as the $S$-duality of Spanier and Whitehead (loc. cit.) and the spectral sequences of Adams [1].

In these circumstances Puppe [17] was led to propose an intrinsic definition of a stable homotopy category as a category with certain additional structure (cf. $\S 9$ below). Stable homotopy categories of spaces are examples of these objects, but others may be derived from algebraic sources, viz. from certain categories of chain complexes. The notion has proved useful and has been exploited both in topological and in algebraic contexts (Heller [14], Verdier [21], Boardman [2], etc).

Freyd is responsible [9] for the observation that Puppe's stable homotopy categories are canonically imbedded in abelian categories or, more precisely, that categories which admit the additional structure described by Puppe are so imbedded. The well-developed methods of homological algebra can thus be directly applied to the study of stable homotopy; Freyd has begun the exploitation of these methods [10], [11].

${ }^{1}$ An address delivered before the Seventy-third Annual Meeting of the Society at Houston on January 26, 1967 by invitation of the Committee to Select Hour Speakers for Annual and Summer Meetings; received by the editors July 17, 1967.

2 This work was in part supported by a grant from the National Science Foundation 
The dichotomy just observed between the underlying category and its additional structure suggests that the terminology of Verdier, which distinguishes between a "triangulable" category and its "triangulations," is to be preferred to that of Puppe. Accordingly, the "stable categories" of Puppe are referred to below as triangulated categories (the word "stable" is itself used in a quite different way, cf. \$1).

Although triangulated categories have had fruitful applications there is reason to believe that they omit a great deal of important information about stable homotopy. One point (which will not be discussed below) is that the standard filtrations of homotopy theory by skeletons, Postnikov systems, Adams resolutions, and so forth, have lost so much structure when looked at in the stable homotopy category that all that is left essentially is their associated spectral sequences. Another (cf. \$17) is that triangulable categories of ten have enormously many triangulations, among which Puppe's axioms do not distinguish.

Verdier (loc. cit.) has discovered a property (the so-called octahedral axiom) of the standard triangulations of stable homotopy categories which appears to be independent of Puppe's axioms but hardly seems adequate to replace the missing structure.

In searching for this missing structure one is led to look again at the several examples of triangulated categories in order to find a common antecedent for the algebraic as well as the topological cases providing a uniform rationale for the construction of stable homotopy categories and thus providing a point at which to begin the search.

To fill this role the notion of an h-c-category is proposed here ( $\$ 3)$ as the appropriate structure in which to do homotopy theory. It should be emphasized that axiomatic homotopy theory, in this sense of homotopy theory in an abstract category, is not new, going back at least to Kan [15]. Most of the previous axiomatizations have depended on some sort of a functorial analogue of the product with the unit interval (cylinder functor) or the join with a point (cone functor). While these seem to deal reasonably well with the topological examples, they are less adapted to the algebraic ones. In any case the present problem seemed to demand a new axiomatization.

In the one we use here, the additional structure consists not in a cylinder or cone functor but in the homotopy relation and the $a$ priori choice of the class of cofibrations. This is precisely what is needed to write down the Eilenberg-Steenrod axioms (without the dimension axiom) for homology theory. It then appears that the canonical functor from the original category to the Freyd imbedding of the stable 
homotopy category is not merely a homology theory but the universal homology theory of the h-c-category. This universal homology theorem (Theorem 10.3 below) asserts, in effect, the inevitability of stable homotopy theory.

The exposition of this argument occupies Chapter I. Chapter II contains: first, applications of the universal homology theorem; second, a discussion of the relation between triangulations and the Toda bracket; third, a computation of the class of triangulations of a triangulable category.

The applications are (\$11) a representability theorem for groupvalued homology and cohomology theories analogous to results of Brown [3], [4], Whitehead [22] (cf. also Dold [6]), and (\$12) a novel proof of a theorem of Dold [5], [6] on homology theories with values in the category of rational vector spaces. These are, so to speak, 0 th order applications, since they make no explicit use of the standard triangulation of the stable homotopy category.

A first order application $(\$ \$ 13,14)$ is to the Toda bracket [20] in the form discussed by Spanier [18]. The notion of a secondary composition can be defined in great generality; the Toda bracket, which is defined in any h-c-category, gives an example. It turns out that the stable Toda bracket can be computed from the standard triangulation of the stable homotopy category.

The computation $(\$ \$ 15-17)$ of the class of triangulations of a triangulable category justifies the assertion that there are in general many triangulations. In particular it is shown that for the stable homotopy category of the category of finite complexes the class is uncountable.

Chapter III is devoted to the proof of the relativization theorem for h-c-categories which asserts that the cofibrations of an h-c-category are the objects of a new h-c-category. For topological spaces it would presumably be well known except for the want of a language in which to state it. For the case of homological algebra, cf. [12].

This relativization theorem implies that to each h-c-category is associated a sequence of stable homotopy categories. No argument will be made here to this effect, but it seems clear that a good deal of the missing information in stable homotopy is given by this sequence of categories and the functors connecting its terms. The study of this structure forms no part of the aims of this paper; it is suggested rather that it may be a useful object of further study.

\section{Chapter I}

1. Stable categories. We shall often be concerned below with categories provided with a functorial endomorphism which, for one reason 
or another, is called "suspension." It will be convenient to allow ourselves to denote suspensions in different categories by the same letter, usually $\Sigma$, and to adopt the verbal device of calling categories so provided "categories with suspensions."

If $\mathfrak{e}, \mathfrak{C}^{\prime}$ are categories with suspensions, a functor $F: \mathfrak{e} \rightarrow \mathfrak{C}^{\prime}$ is stable if $F \Sigma=\Sigma F$, and weakly stable if $F \Sigma \approx \Sigma F$.

If $\mathfrak{C}$ has a suspension, a triangle in $\mathcal{C}$ is a diagram $A \rightarrow B \rightarrow C \rightarrow \Sigma A$; a morphism of triangles is a commutative diagram

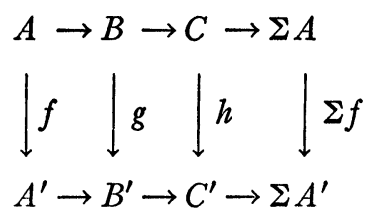

and the category $\operatorname{Tr} \mathfrak{C}$ of triangles in $\mathcal{C}$ is defined accordingly. If $F: \mathfrak{C} \rightarrow \mathfrak{C}^{\prime}$ is a stable functor, it clearly induces a functor $\operatorname{Tr} \mathfrak{e} \rightarrow \operatorname{Tr} \mathfrak{C}^{\prime}$; if it is weakly stable, it is easy to manufacture, for each isomorphism $\Sigma F \approx F \Sigma$, such a functor.

We shall say that a category $\mathfrak{e}$ with a suspension is stable if the suspension is an automorphism of $\mathfrak{C}$. Any category with suspension has associated to it a "universal" stable category, by the following construction.

If $\mathfrak{C}$ is a category with suspension, set ob $\mathfrak{S C}=o b \mathfrak{e} \times \boldsymbol{Z}$ and define

$$
\operatorname{se}((X, m),(Y, n))=\operatorname{inj}_{\boldsymbol{k}} \lim \mathrm{e}\left(\Sigma^{k+m} X, \Sigma^{k+n} Y\right) \text {. }
$$

If $i_{k}=i_{k}(X, m, Y, n): \mathfrak{e}\left(\Sigma^{k+m} X, \Sigma^{k+n} Y\right) \rightarrow \mathcal{S e}((X, m),(Y, n))$ is the injection, the composition in $s \mathfrak{e}$ is defined by

$$
\left(i_{k} g\right)\left(i_{k} f\right)=i_{k}(g f),
$$

$k$ being taken sufficiently large; this is of course independent of the several choices. Se is easily seen to be a category. Its suspension $\Sigma$ is given by $\Sigma(X, m)=(X, m+1)$ on objects and $\Sigma i_{k}(X, m, Y, n) f$ $=i_{k-1}(X, m+1, Y, n+1) f$ on morphisms.

We may define a functor $S: \mathbb{e} \rightarrow \mathfrak{S e}$ by $S X=(X, 0)$ and $S f=i_{0} f$; we shall identify $X \in \mathcal{C}$ with $(X, 0) \in \mathcal{S}$ so that ob $\mathcal{C} \subset$ ob $s \mathcal{C}$. Further, we may define an isomorphism $\sigma: S \Sigma \approx \Sigma S$ by $\sigma_{X}=i_{0}(\Sigma X, 0, X, 1) 1_{\Sigma X}$, its inverse being $\sigma_{\overline{\mathbf{X}}}^{1}=i_{0}(X, 1, \Sigma X, 0) 1_{\Sigma X}$, so $S$ is weakly stable.

The universality of $\mathcal{S C}$ is expressed by the following statement.

Proposition 1.1. If $a$ is a stable category, $F: \mathrm{e} \rightarrow Q$ is weakly stable, and $\theta: F \Sigma \approx \Sigma F$, then there is a unique stable functor $G: S \mathrm{e} \rightarrow Q$ with $G S=F, G \sigma=\theta$. 
We must define $G(X, m)=\Sigma^{m} F X$; for $f: \Sigma^{k+m} X \rightarrow \Sigma^{k+n} Y$, we set $G\left(i_{k} f\right)=\Sigma^{-k} \phi$ where

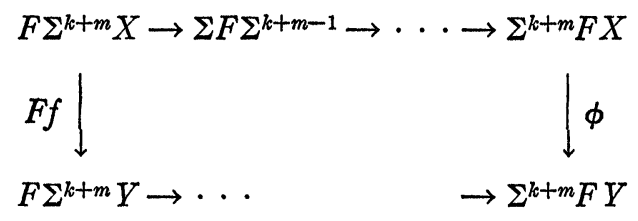

commutes, the horizontal arrows being suitable values of $\theta$.

In virtue of this universality we may refer to $\mathcal{S C}$ as the stabilization of $\mathfrak{e}$.

2. Homology theories. The domain of a homology theory is a category $\mathfrak{C}$ supplied with certain additional structure, viz. a congruence $\simeq$, called homotopy, and a collection of sequences $X=\left(X^{\prime} \stackrel{x^{\prime \prime}}{\rightarrow} X \stackrel{x^{\prime}}{\rightarrow} X^{\prime \prime}\right)$. It is convenient to regard these sequences as the objects of a full subcategory cof $\mathfrak{e}$ of the appropriate category of diagrams. The functors $T^{\prime}, T, T^{\prime \prime}:$ cof $\mathrm{e} \rightarrow \mathfrak{C}$ are defined by $X \mapsto X^{\prime}, X, X^{\prime \prime}$ and the morphisms $T^{\prime \prime} \stackrel{\prime^{\prime \prime}}{\rightarrow} T \stackrel{t^{\prime}}{\rightarrow} T^{\prime \prime}$ by $X \mapsto x^{\prime \prime}, x^{\prime}$.

The range of a homology theory will always be taken to be a stable abelian category: notice that the suspension in such a category is an exact functor. We shall say that a triangle $A \rightarrow B \rightarrow C \rightarrow \Sigma A$ is exact whenever it is exact not merely as a diagram but also "at $A$," i.e. when

$$
\cdots \rightarrow \Sigma^{-1} C \rightarrow A \rightarrow B \rightarrow C \rightarrow \Sigma A \rightarrow \Sigma B \rightarrow \cdots
$$

is exact.

If $\mathcal{C}$ is a suitable domain and $Q$ is stable abelian, a homology theory $(h, \partial)$ from $\mathfrak{C}$ to $Q$ consists of a functor $h: \mathfrak{C} \rightarrow \mathbb{Q}$ and a morphism of functors $\partial: h T^{\prime \prime} \rightarrow \Sigma h T^{\prime}$ satisfying the following two conditions:

Номотору Ахгом. $f \simeq g$ implies $h f=h g$.

Exactness Axiom. If $X \in \operatorname{cof} \mathfrak{e}$, then

$$
h T^{\prime} X \stackrel{h t^{\prime \prime}}{\longrightarrow} h T X \stackrel{h t^{\prime} \mathrm{x}}{\longrightarrow} h T^{\prime \prime} X \stackrel{\partial \mathrm{x}}{\longrightarrow} \Sigma h T^{\prime} X
$$

is an exact triangle in $Q$.

$\partial$ is of course the connecting morphism of the homology theory; we shall of ten use the same symbol for the connecting morphisms of different theories and, elliptically, write $h$ instead of $(h, \partial)$ for the homology theory.

If $h, h^{\prime}$ are both homology theories from $\mathcal{C}$ to $\mathfrak{a}$, a morphism of homology theories $(h, \partial) \rightarrow\left(h^{\prime}, \partial\right)$ is a morphism $\phi: h \rightarrow h^{\prime}$ of functors such that 


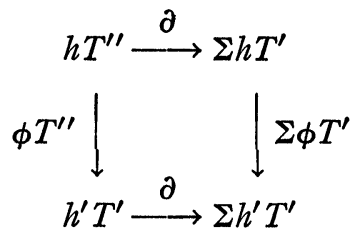

commutes. The category of such homology theories is defined accordingly.

The following assertion is self-evident:

Proposition 2.1. Let $h$ be a homology theory from e to $a$. Suppose $\mathfrak{C}^{\prime}$ is a suitable domain for a homology theory and that $G: \mathfrak{C}^{\prime} \rightarrow \mathfrak{C}$ preserves homotopy and takes cof $\mathfrak{C}^{\prime}$ into cof $\mathfrak{e}$. Then $(h G, \partial G)$ is again a homology theory. Suppose on the other hand that $F: Q \rightarrow Q^{\prime}$ is a stable exact functor. Then $(F h, F \partial)$ is also a homology theory.

The latter observation suggests the definition of a universal homology theory on a category $\mathfrak{e}$, viz. a homology theory $h$ from $\mathfrak{e}$ to, say, $H \mathcal{C}$, such that for any homology theory $h$ from $\mathcal{C}$ to any $Q$ there exists a stable exact functor $F: H \mathfrak{C} \rightarrow Q$ unique up to isomorphism such that $(h, \partial) \approx(F h, F \partial)$.

THEOREM 2.2. If $\mathrm{e}$ is a small category provided with a congruence $\cong$ and a full subcategory cof $\mathrm{e}$ of sequences $X^{\prime} \rightarrow X \rightarrow X^{\prime \prime}$, then there exists a universal homology theory on $\mathrm{e}$.

The functorial character of the value category $H \mathcal{C}$ and the naturality of the homology theory follow immediately.

We shall not use this theorem below; we thus content ourselves with a brief outline of the proof.

(2.3) If $h$ is a homology theory from $\mathcal{C}$ to $a$, then there is a smallest exact stable subcategory $Q^{\prime}$ of $Q$ through which $h$ and $\partial$ factor. Call this the subcategory generated by $h$. All such subcategories have cardinality bounded by a cardinal depending only on $\mathfrak{e}$.

(2.4) Thus there is a family of homology theories $h_{\alpha}: \mathfrak{e} \rightarrow \mathfrak{Q}_{\alpha}$ indexed by a set such that any homology theory is of the form $\left(F h_{\alpha}, F \partial_{\alpha}\right)$ with $F$ stable exact.

(2.5) The functor $h^{*}: \mathfrak{e} \rightarrow \prod_{\alpha} Q_{\alpha}$ and the morphism $\partial^{*}$ with projections $h_{\alpha}, \partial_{\alpha}$ are a homology theory. If the values are restricted to the subcategory generated by $h^{*}$, it becomes universal.

3. h-c-categories. Since our concern here is with stable homotopy we must introduce notions of structure in a category which allow us to make enough of the constructions of homotopy theory to re- 
produce the results of Puppe [17] and Freyd [9].

We restrict our attention to categories with a 0 -object. In such a category $\mathfrak{e}$, a system of cofibrations is a family of morphisms which we regard as the objects of a full subcategory Cof $\mathbb{e}$ of the morphismcategory of $\mathfrak{C}$ satisfying the following conditions:

(C0) Cof $\mathfrak{e}$ is replete, contains all morphisms $0 \rightarrow A$ and all identity morphisms, and is closed under composition.

(C1) If $\left(A^{\prime} \stackrel{a^{\prime}}{\rightarrow} A\right) \in$ Cof $\mathcal{C}$ and $A^{\prime} \stackrel{\prime^{\prime}}{\rightarrow} B^{\prime}$ is any morphism, then there is a pushout diagram (=cocartesian square).

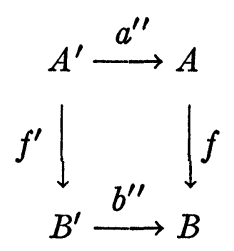

and $b^{\prime \prime}$ is once more in Cof $\mathfrak{e}$.

In particular, taking $A^{\prime}=0$, we see that $\mathcal{C}$ has finite coproducts; we use the notation $B^{\prime} \vee A$. More generally we write $B=B^{\prime} \cup A$ for the pushout when there is no confusion possible as to the morphisms $a^{\prime \prime}, f^{\prime}$. We also generalize the matrix notation, familiar for coproducts, as follows. If in the diagram 3.1 above $\beta: B^{\prime} \rightarrow X$ and $\alpha: A \rightarrow X$ satisfy $\beta f^{\prime}=\alpha a^{\prime \prime}$, then $(\beta \alpha): B^{\prime} \cup A \rightarrow X$ is the unique morphism with $(\beta \alpha) b^{\prime \prime}=\beta,(\beta \alpha) f=\alpha$.

Taking $B^{\prime}=0$ in $3.1, A \stackrel{f}{\rightarrow} B$ becomes the cokernel of $a^{\prime \prime}$. We call $f$ (or sometimes $B$ ) the cofibre of the cofibration $a^{\prime \prime}$ and occasionally write $A / A^{\prime}$ for $B$. We shall further interpret the statement " $A^{\prime \prime} \stackrel{\text { " }}{\rightarrow} A$ $\stackrel{a^{\prime}}{\rightarrow} A^{\prime \prime}$ is a cofibration" to mean that $a^{\prime \prime}$ is a cofibration and $a^{\prime}$ is its cofibre. The full category of diagrams whose objects are cofibrations in this sense is cof $\mathfrak{e}$. The forgetful functor cof $\mathfrak{e} \rightarrow \operatorname{Cof} \mathfrak{e}$ is of course an equivalence of categories.

A category provided with a system of cofibrations is a c-category. It is an h-c-category if it is further provided with a congruence $\simeq$, called homotopy, satisfying four additional conditions. Before listing these we introduce the following terminology. The quotient category $\mathfrak{e}^{\square}=\mathfrak{e} / \simeq$ is the homotopy category of $\mathfrak{e}$; a morphism in $\mathfrak{C}$ is a homotopy equivalence if its image in $\mathrm{e}^{\square}$ is an isomorphism; an object $A$ of $\mathcal{C}$ is contractible if $A \rightarrow 0$ is a homotopy equivalence.

The axioms for an h-c-category are the following:

(HC 1) (Additivity) If $f \simeq f^{\prime}: A \rightarrow X$ and $g \simeq g^{\prime}: B \rightarrow X$, then $(f g$ ) $\simeq\left(f^{\prime} g^{\prime}\right): A \bigvee B \rightarrow X$.

(HC 2) (Homotopy extension) If $\left(f^{\prime}, f, f^{\prime \prime}\right)$ is a morphism in cof $C$ 
and $g^{\prime} \simeq f^{\prime}$, then there are morphisms $g \simeq f, g^{\prime \prime} \simeq f^{\prime \prime}$ such that $\left(g^{\prime}, g, g^{\prime \prime}\right)$ is also a morphism in cof $\mathbb{C}$.

(HC 3) (Deformation retraction) If, in the pushout diagram 3.1, the morphism $a^{\prime \prime}$ is a homotopy equivalence as well as a cofibration, then so is $b^{\prime \prime}$.

(HC 4) (Mapping cylinder) There exists a function which assigns to every morphism $h$ in $\mathfrak{e}$ a mapping cylinder, i.e. a factorization $h=g f$ with $g$ a homotopy equivalence and $f$ a cofibration.

The rather stilted form of the last axiom is needed only if we are interested in large categories. If $\mathfrak{e}$ is small, all that is being asserted is the existence of a mapping cylinder for each $h$.

Notice that an h-c-category $\mathcal{C}$, supplied with the additional structure $\simeq$ and cof $\mathfrak{e}$, is an appropriate domain for homology theories.

4. Some examples of h-c-categories. We list here a variety of examples. In no case do we prove that the axioms are satisfied; indeed their assertions are generally either well-known facts or easy exercises. An occasional vagueness is intentional; it is meant to suggest that any one of several interpretations would yield an h-c-category.

(4.1) Let $\mathcal{C}$ be a suitable category of well-pointed topological spaces, i.e. spaces with basepoints whose inclusion satisfies the homotopy extension condition; morphisms are basepoint-preserving maps. Cofibrations are maps satisfying the homotopy-extension condition, and $\simeq$ means homotopy with basepoints fixed.

(4.2) Fix a group $G$. Let $\mathcal{C}$ have as objects $G$-spaces with fixed basepoint whose inclusion satisfies the obvious equivariant homotopy-extension condition. Morphisms are basepoint-preserving equivariant maps. Cofibrations are defined by the equivariant homotopy-extension property, and $\simeq$ means equivariant homotopy with basepoints fixed.

(4.3) $\mathcal{C}$ is the dual of a suitable category of topological spaces with basepoints. Homotopy has its usual sense. A cofibration in $\mathbb{C}$ is a fibration in the category of topological spaces.

(4.4) Take for $\mathcal{C}$ an additive category with a suitable "relative" abelian structure (cf. [7], [12]) with enough injectives. Cofibrations are proper monomorphisms of the abelian structure. Two morphisms are homotopic if their difference can be factored through an injective.

Categories of chain complexes, such as those considered by Puppe [17], are included under this last heading. Suitable categories of spectra may also be regarded as h-c-categories. The descriptions are more complicated and we omit them as not pertinent to our interests here. 
We shall exhibit below an additional class of examples: the cofibrations in an h-c-category again form an h-c-category. We shall suggest indeed that this fact is central to the full understanding of stable homotopy.

5. The suspension functor. In $\S \S 5-10$ we shall be working with an h-c-category e.

Lemma 5.1. If $A^{\prime a^{\prime \prime}} \rightarrow A \stackrel{a^{\prime}}{\rightarrow} A^{\prime \prime}$ is a cofibration and $A^{\prime}$ is contractible then $a^{\prime}$ is a homotopy equivalence.

For by the homotopy extension axiom it has an endomorphism $\left(0, f, f^{\prime \prime}\right)$ in cof $\mathcal{e}$ with $f \simeq 1_{A}, f^{\prime \prime} \simeq 1_{A^{\prime \prime}}$. But $f a^{\prime \prime}=0$ so that $f=g a^{\prime}$, $a^{\prime} g a^{\prime}=f^{\prime \prime} a^{\prime}, a^{\prime} g=f^{\prime \prime}$, and $g$ is thus a homotopy inverse of $a^{\prime}$.

A cone over $A$ is a cofibration $A \rightarrow C A$ with $C A$ contractible. The mapping cylinder axiom, applied to $A \rightarrow 0$, shows their existence. A mapping cone of a cofibration $A \rightarrow X \stackrel{\eta}{\rightarrow} X / A$ is a pushout $C A \cup X$ where $A \rightarrow C A$ is a cone over $A$; its existence is guaranteed by the pushout axiom $\mathrm{C} 1$ as is the fact that $C A \rightarrow C A \cup X \stackrel{(0 \eta)}{\rightarrow} X / A$ is a cofibration. From (5.1) it follows that $(0 \eta)$ is a homotopy equivalence.

Lemma 5.2. If $A^{\prime} \stackrel{a^{\prime \prime}}{\rightarrow} A \stackrel{a^{\prime}}{\rightarrow} A^{\prime \prime}$ and $B^{\prime} \stackrel{b^{\prime \prime}}{\rightarrow} B \stackrel{b^{\prime}}{\rightarrow} B^{\prime \prime}$ are cofibrations, $B$ is contractible, and $f^{\prime}: A^{\prime} \rightarrow B^{\prime}$, then there is a morphism $\left(f^{\prime}, f, f^{\prime \prime}\right)$ of the cofibrations. If $\left(g^{\prime}, g, g^{\prime \prime}\right)$ is another, and $g^{\prime} \simeq f^{\prime}$, then $g^{\prime \prime} \simeq f^{\prime \prime}$.

The first statement follows immediately from the homotopy extension axiom applied to the morphism $(0,0,0)$ into the cofibration $B \rightarrow B \rightarrow 0$ since $b^{\prime \prime} f^{\prime} \simeq 0$.

For the second we may, again in virtue of the homotopy extension axiom, assume without loss of generality that $f^{\prime}=g^{\prime}$. Now consider the commutative diagrams

$$
\begin{aligned}
& f^{\prime}=g^{\prime} A^{\prime} \longrightarrow C A^{\prime} \stackrel{\alpha}{\longrightarrow} C A^{\prime} / A^{\prime} \\
& B^{\prime} \longrightarrow C B^{\prime} \stackrel{\beta}{\longrightarrow} C B^{\prime} / B^{\prime},
\end{aligned}
$$

whose existence is guaranteed by the previous argument, and

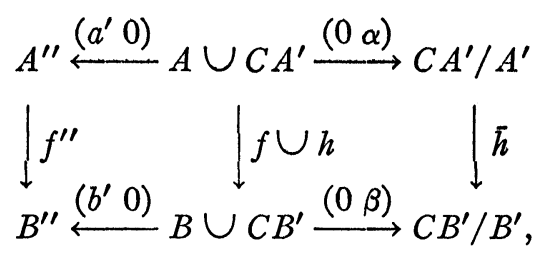


as well as the similar one with $g^{\prime \prime}, g \cup h$ substituted for $f^{\prime \prime}, f \cup h$. If we project these in to $\mathrm{e}^{\square}$ and indicate the homotopy class of a morphism by the same expression as the morphism itself, then $(0 \beta)$ is an isomorphism and $f^{\prime \prime}=\left(\begin{array}{ll}b^{\prime} & 0\end{array}\right)\left(\begin{array}{lll}0 & \beta\end{array}\right)^{-1} h\left(\begin{array}{ll}0 & \alpha\end{array}\right)=g^{\prime \prime}$.

Proposition 5.3. For each $A \in \mathcal{C}$ let $A \rightarrow C A$ be a cone over $A$ with cofibre $\Sigma A$. Then a functor $\Sigma: \mathfrak{e}^{\square} \rightarrow \mathfrak{e}^{\square}$ is defined by the condition that for any $\phi \in \mathbb{C}^{\square}(A, B)$ there be a morphism $\left(f^{\prime}, f, f^{\prime \prime}\right)$ of $A \rightarrow C A \rightarrow \Sigma A$ into $B \rightarrow C B \rightarrow \Sigma B$ such that $f^{\prime} \in \phi, f^{\prime \prime} \in \Sigma \phi$. If $\left\{A \rightarrow C^{\prime} A \rightarrow \Sigma^{\prime} A\right\}$ is another family of cones, then the functors $\Sigma, \Sigma^{\prime}$ are canonically isomorphic.

We shall speak of any one of these $\Sigma$ as the suspension functor in $\mathrm{e}^{\square}$.

We shall see that any homology theory on $\mathcal{C}$ is weakly stable with respect to this suspension. Suppose $(h, \partial)$ is a homology theory from $\mathcal{e}$ to a stable abelian category $B$. Since $0 \rightarrow 0 \rightarrow 0$ is a cofibration, $h 0=0$ and thus $h$ vanishes on contractible objects. But for any $A \in \mathrm{C}$ the cofibration $A \rightarrow C A \rightarrow \Sigma A$ gives an exact sequence $h C A \rightarrow h \Sigma A$ $\stackrel{\partial}{\rightarrow} \Sigma h A \rightarrow \Sigma h C A$ so that $\partial: h \Sigma A \simeq \Sigma h A$. The inverse isomorphism we may write $\theta_{A}: \Sigma h A \rightarrow h \Sigma A$. But by the homotopy axiom $h$ factors through $\mathfrak{e}^{\square}$. If we write $\mathrm{C} \rightarrow \mathrm{e}^{\square} \stackrel{\bar{h}}{\rightarrow} \cap$ for the factorization we have proved the following assertion.

LEMMA 5.4. $\theta: \Sigma \bar{h} \approx \bar{h} \Sigma$ makes $\bar{h}$ a weakly stable functor $\bar{h}: \mathrm{e}^{\square} \rightarrow ß$.

6. Cylinders. A cylinder over $A$ is a cofibration $j=\left(j_{0} j_{1}\right): A \bigvee A$ $\rightarrow Z A$ such that $j_{0}, j_{1}$ are homotopic and are homotopy equivalences. A morphism $p: Z A \rightarrow A$ is a projection of the cylinder if $p j_{0}=p j_{1}=1_{A}$.

Proposition 6.1. Every cylinder has a projection; any two projections are homotopic. Every object has a cylinder. If $\left(j_{0} j_{1}\right): A \rightarrow Z A$ is a cylinder then $f_{0}, f_{1}: A \rightarrow X$ are homotopic if and only if there is an $F: Z A \rightarrow X$ such that $F j_{0}=f_{0}, F j_{1}=f_{1}$.

The first assertion follows easily from the homotopy extension axiom. The existence of cylinders is guaranteed by the mapping cylinder axiom applied to the folding map (1 1$): A \vee A \rightarrow A$. For the last, let $p$ be a projection. Then $f_{0} p: Z A \rightarrow X$ and $f_{0} p\left(j_{0} j_{1}\right)=\left(f_{0} f_{0}\right)$ $\approx\left(f_{0} f_{1}\right)$ by the additivity axiom. The existence of $F$ follows from the homotopy extension axiom.

If $\left(j_{0} j_{1}\right): A \rightarrow Z A$ is a cylinder, then $j_{0}, j_{1}$ are cofibrations. It follows immediately from the deformation-retraction axiom that the cofibre $C_{i} A$ of $j_{i}$ is contractible. But the diagram 


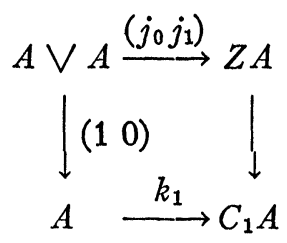

is a pushout. Thus $k_{i}$ is a cone over $A$. We may thus construct a commutative diagram

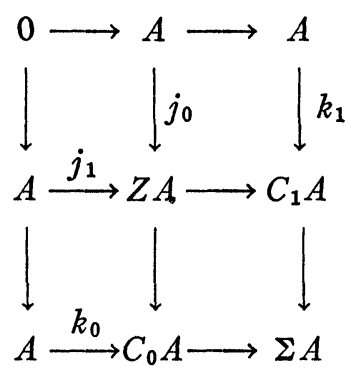

in which all rows and columns are cofibrations. The morphism $Z A \rightarrow \Sigma A$ is the cofibre of $\left(j_{0} j_{1}\right)$. With respect to either of the cones $C_{i} A, \Sigma A$ is of course the suspension of $A$.

LEMMA 6.3. If $\left(j_{0} j_{1}\right): A \bigvee A \rightarrow Z A$ and $\left(j_{0}^{\prime} j_{1}^{\prime}\right): A^{\prime} \bigvee A^{\prime} \rightarrow Z A^{\prime}$ are cylinders and $f_{0} \simeq f_{1}: A \rightarrow A^{\prime}$, then there is a commutative diagram

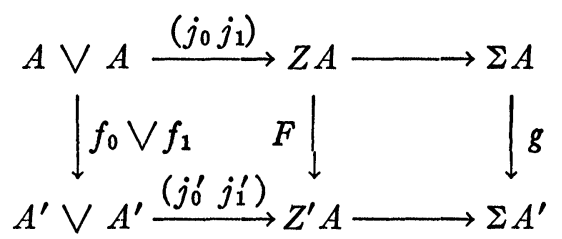

For any such diagram the homotopy class of $g$ is the suspension of that of the $f_{i}$.

Since $j_{0}^{\prime} f_{0} \simeq j_{1}^{\prime} f_{1}$, the existence of $F$ follows from Proposition 6.1. We may use it to construct a morphism of the diagrams (6.2); the second conclusion follows immediately.

As in conventional homotopy theory we shall want to attach cylinders end-to-end. If $\left(j_{0} j_{1}\right): A \bigvee A \rightarrow Z A$ and $\left(j_{0}^{\prime} j_{1}^{\prime}\right): A \bigvee A \rightarrow Z^{\prime} A$ are cylinders, we consider the pushout diagrams

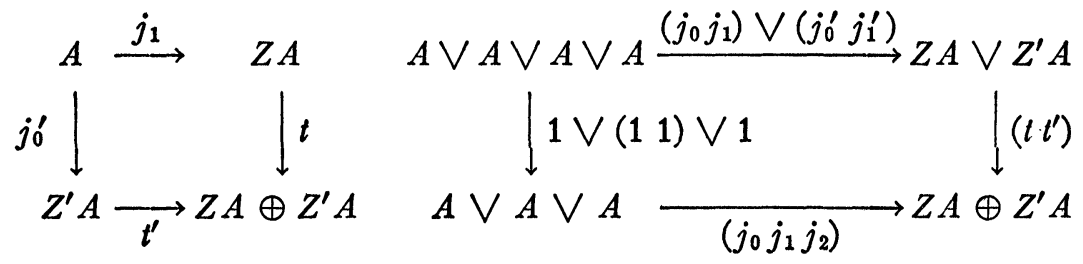


where we have allowed ourselves to write, in the bottom row of the latter, $j_{0}$ for $t j_{0}, j_{1}$ for $t j_{1}=t^{\prime} j_{0}^{\prime}$, and $j_{2}$ for $t^{\prime} j_{1}^{\prime}$. By the deformationretraction axiom $t$ and $t^{\prime}$ are homotopy equivalences, thus also $j_{0}, j_{1}$, $j_{2}$; and $\left(j_{0} j_{2}\right): A \bigvee A \rightarrow Z A \oplus Z^{\prime} A$ is again a cylinder over $A$. We define analogously cylinders $\left(j_{0} j_{3}\right): A \bigvee A \rightarrow Z A \oplus Z^{\prime} A \oplus Z^{\prime \prime} A$.

Starting with a cylinder $\left(j_{0} j_{1}\right): A \bigvee A \rightarrow Z A$, we have the following cofibrations:

$$
\begin{gathered}
A \vee A \stackrel{\left(j_{0} j_{1}\right)}{\longrightarrow} Z A \longrightarrow \Sigma A \\
A \vee A \stackrel{\left(j_{0} j_{2}\right)}{\longrightarrow} Z A \oplus Z A \longrightarrow \Sigma^{(2)} A \\
A \vee A \stackrel{\left(j_{0} j_{3}\right)}{\longrightarrow} Z A \oplus Z A \oplus Z A \longrightarrow \Sigma^{(3)} A \\
A \vee A \vee A \stackrel{\left(j_{0} j_{1} j_{2}\right)}{\longrightarrow} Z A \oplus Z A \longrightarrow \Sigma A \vee \Sigma A \\
A \vee A \vee A \stackrel{\left(j_{0} j_{1} j_{3}\right)}{\longrightarrow} Z A \oplus Z A \oplus Z A \longrightarrow \Sigma A \vee \Sigma^{(2)} A \\
A \vee A \vee A \stackrel{\left(j_{0} j_{2} j_{3}\right)}{\longrightarrow} Z A \oplus Z A \oplus Z A \longrightarrow \Sigma^{(2)} A \vee \Sigma A,
\end{gathered}
$$

where $\Sigma^{(2)} A, \Sigma^{(3)} A$ are defined to be the appropriate cofibres. We shall want to introduce the following (temporary) notation. If a morphism $f$ of the middle terms of any of the cofibrations above induces a morphism of cofibrations, we write $\langle f\rangle$ for the induced morphism on the cofibre. For example, if $p$ is a projection of the original cylinder, we have

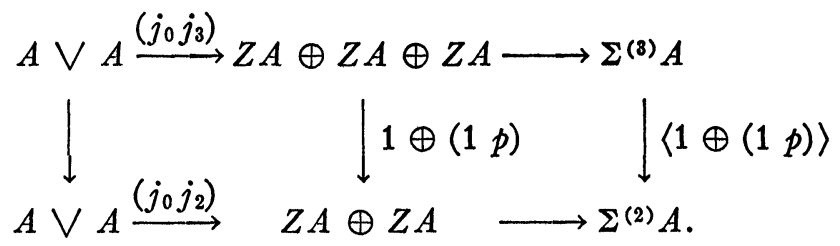

7. The cogroup structure of $\Sigma$. We suppose that we have chosen for each $A \in \mathcal{C}$ a cylinder $\left(j_{0} j_{1}\right): A \bigvee A \rightarrow Z A$ thus defining a suspension functor $\Sigma: \mathfrak{e}^{\square} \rightarrow \mathfrak{e}^{\square}$. It follows immediately from Lemma 6.3 that the morphisms $\left\langle\left(\begin{array}{ll}1 & p\end{array}\right)\right\rangle,\left\langle\left(\begin{array}{ll}p & 1\end{array}\right)\right\rangle: \Sigma^{(2)} A \rightarrow \Sigma A$ are homotopy equivalences. If we allow a morphism in $\mathfrak{e}$ to stand for its image in $\mathfrak{e}^{\square}$, i.e. its homotopy class, we may define $\mu_{A}: \Sigma A \rightarrow \Sigma A \bigvee \Sigma A$ in $\mathfrak{e}^{\square}$ as the composition

$$
\Sigma A \stackrel{\langle(p 1)\rangle^{-1}=\langle(1 p)\rangle^{-1}}{\longrightarrow} \Sigma^{(2)} A \stackrel{\left\langle 1_{z A} \oplus z_{\Lambda}\right\rangle}{\longrightarrow} \Sigma A \bigvee \Sigma A .
$$


Also, $\left(j_{1} j_{0}\right): A \bigvee A \rightarrow Z A$ is again a cylinder with the same cofibre $\Sigma A$. Thus by Lemma 6.3 there is an $F: Z A \rightarrow Z A$ with $F j_{i}=j_{1-i}$. The homotopy class of $\langle F\rangle: \Sigma A \rightarrow \Sigma A$ is $\iota_{A}$.

THEOREM 7.1. $\mu: \Sigma \rightarrow \Sigma \bigvee \Sigma$ and $\iota: \Sigma \rightarrow \Sigma$ are natural transformations giving to $\Sigma$ the structure of a cogroup.

The naturality follows from Lemma 6.3. We recall that the latter assertion is equivalent to the statement that for each $A \mu_{A}: \Sigma A \rightarrow$ $\Sigma A \bigvee \Sigma A, \iota_{A}: \Sigma A \rightarrow \Sigma A$ give $\Sigma A$ the structure of a cogroup.

The counit property of $\mu$ is the assertion that the composition $\Sigma \stackrel{\mu}{\rightarrow} \Sigma \vee \Sigma^{(10)} \Sigma$ is the identity in $\mathrm{e}^{\square}$. But the composition $\Sigma^{(2)} A$ $\stackrel{\langle 1\rangle}{\rightarrow} \Sigma A \vee \Sigma A \stackrel{(10)}{\rightarrow} \Sigma A$ is just $\langle(1 p)\rangle$.

For the associativity of $\mu$ we consider the following commutative diagram,

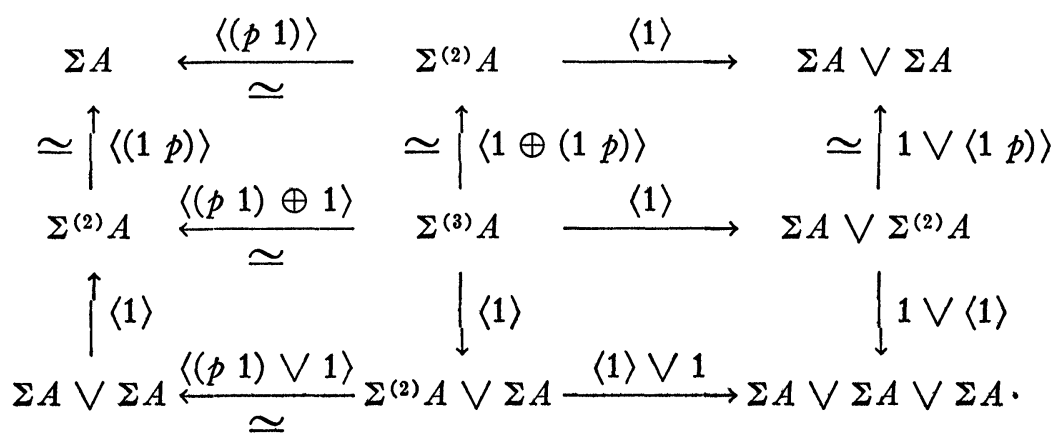

It follows from Lemma 6.3 that the morphisms labeled " $\simeq$ " are homotopy equivalences. But the inverse composites (in $\mathrm{e}^{\square}$ ) along the outside edges then give $\left(1 \vee \mu_{A}\right) \mu_{A}=\left(\mu_{A} \vee 1\right) \mu_{A}$

The fact that $\iota$ is an inverse for the comultiplication $\mu$ follows from the commutativity of the diagram

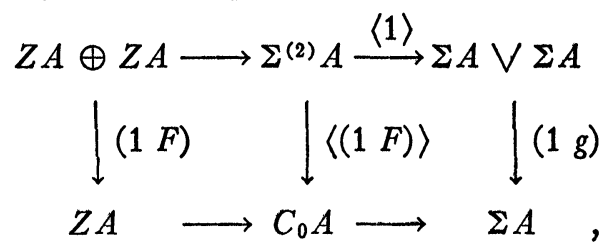

which implies that $\left(\begin{array}{ll}1 & \iota\end{array}\right)\langle 1\rangle\left\langle\left(\begin{array}{ll}p & 1\end{array}\right)\right\rangle^{-1}=\left(\begin{array}{ll}1 & \iota\end{array}\right) \mu=0$ in $\mathrm{e}^{\square}$.

Using a well-known result of categorical algebra we deduce the following corollary.

CoROllary 7.2. $\Sigma \mu=\mu \Sigma: \Sigma^{2} \rightarrow \Sigma^{2} \bigvee \Sigma^{2}, \Sigma_{\iota}=\iota: \Sigma^{2} \rightarrow \Sigma^{2}$. These morphisms give to $\Sigma^{2}$ the structure of an abelian cogroup. 
Since, for each $A \in \mathbb{C}^{\square}, \Sigma A$ is a cogroup, $\mathfrak{e}^{\square}(\Sigma A, B)$ is always a group. We write these groups additively even when they are not commutative. Thus $(f g) \mu_{A}=f+g, f \iota_{A}=-f$.

It is often more convenient to work with cones rather than cylinders in defining the suspension functor. The reader will readily verify the following computation rules.

Proposition 7.3. If $\{A \rightarrow C A \stackrel{\alpha}{\rightarrow} \Sigma A\}$ is a system of cones in $\mathfrak{C}$, then $(\alpha 0),(0 \alpha): C A \cup C A \rightarrow \Sigma A$ are homotopy equivalences. The compositions (in $\left.\mathrm{e}^{\square}\right)(0 \alpha)(\alpha)^{-1},(\alpha \quad 0)(0 \alpha)^{-1}$ are both $\iota_{A}$. The composition $\left(\begin{array}{lll}\alpha & \alpha\end{array}\right)\left(\begin{array}{lll}0 & \alpha\end{array}\right)^{-1}$ is $\left(1_{A} \iota_{A}\right) \mu_{A}$. Thus $\mu_{A}=\left(1 \bigvee\left(\begin{array}{lll}0 & \alpha\end{array}\right)\left(\begin{array}{lll}\alpha & 0)^{-1}(\alpha & \alpha\end{array}\right)\left(\begin{array}{ll}0 & \alpha)^{-1}\end{array}\right)\right.$. If $f, g: \Sigma A \rightarrow B$, then $f-g=(f \alpha g \alpha)(\alpha 0)^{-1}$.

8. Puppe sequences. We parallel here the argument of [16]. Compare also [13].

If $A^{\prime \prime} \stackrel{a^{\prime \prime}}{\rightarrow} A \stackrel{a^{\prime}}{\rightarrow} A^{\prime \prime}$ is a cofibration, then $\left(a^{\prime} 0\right): A \cup C A^{\prime} \rightarrow A^{\prime \prime}$ is by Lemma 5.1 a homotopy equivalence and $A \rightarrow A \cup C A^{\prime(0 \alpha)} \stackrel{\rightarrow}{\rightarrow} \Sigma A^{\prime}$ is a cofibration. In $\mathbb{C}^{\square}$ we define $\delta: A^{\prime \prime} \rightarrow \Sigma A^{\prime}$ as $\left(\begin{array}{lll}0 & \alpha\end{array}\right)\left(a^{\prime} \quad 0\right)^{-1}$.

Proposition 8.1. $\delta$ is a natural transformation between the compositions

$$
\operatorname{cof} \mathfrak{e} \stackrel{T^{\prime \prime}}{\rightarrow} \mathfrak{e} \rightarrow \mathfrak{e}^{\square} \quad \text { and } \quad \text { cof } \mathfrak{e} \stackrel{T^{\prime}}{\rightarrow} \mathfrak{e} \rightarrow \mathfrak{e}^{\square} \stackrel{\Sigma}{\rightarrow} \mathfrak{e}^{\square}
$$

This follows immediately from Lemma 5.2.

The morphism $\delta$ is the connecting morphism of the cofibration. With it we construct the triangle $A^{\prime} \rightarrow A \rightarrow A^{\prime \prime} \stackrel{\delta}{\rightarrow} \Sigma A^{\prime}$ in $\operatorname{Tr} \mathrm{e}^{\square}$. The full replete subcategory of $\operatorname{Tr} \mathfrak{e}^{\square}$ generated by these triangles is $\mathrm{h}$-cof $\mathfrak{e}$; its objects are homotopy cofibrations.

Puppe's theorem on homotopy cofibrations is the following assertion.

THEOREM 8.2. If $A^{\prime a^{\prime \prime}} \rightarrow \stackrel{a^{\prime}}{\rightarrow} A^{\prime \prime} \stackrel{a}{\rightarrow} \Sigma A^{\prime}$ is a homotopy cofibration then so is $A \stackrel{a^{\prime}}{\rightarrow} A^{\prime \prime} \stackrel{a}{\rightarrow} \Sigma A^{\prime-\Sigma a^{\prime \prime}} \stackrel{\rightarrow}{\rightarrow} A$.

Notice that $-\Sigma a^{\prime \prime}$ is defined because $\Sigma A^{\prime}$ is a cogroup. What we must show is that the connecting morphism of the cofibration $A \rightarrow A \cup C A^{\prime} \rightarrow \Sigma A^{\prime}$ is $-\Sigma a^{\prime \prime}$. If we make

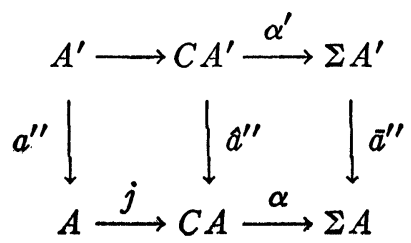


commute, so that $\bar{a}^{\prime \prime}$ represents $\Sigma a^{\prime \prime}$, we may construct in $\mathcal{C}$ the commutative diagram

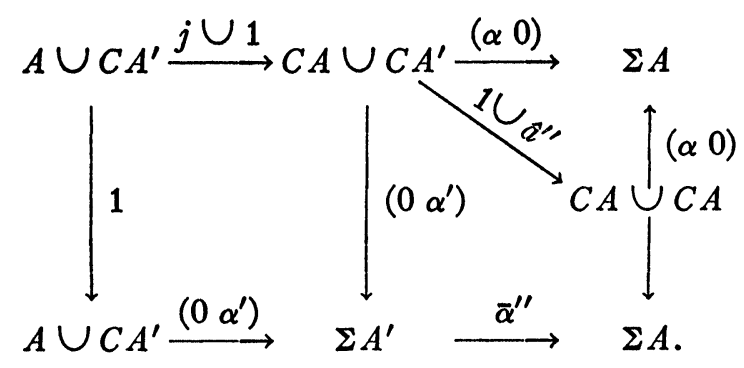

The conclusion then follows using Proposition 7.3.

We record for use below the following easy consequence of the homotopy extension axiom.

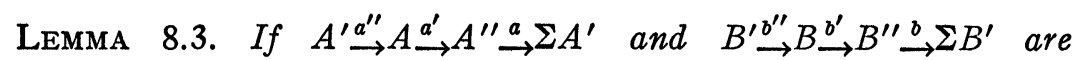
homotopy cofibrations, $f^{\prime}: \overrightarrow{A^{\prime} \rightarrow B^{\prime}, f: A \rightarrow B}$ in $\overrightarrow{\mathrm{e}^{\square}}$, and $\vec{b}^{\prime \prime} f^{\prime}=f a^{\prime \prime}$, then there is a morphism $\left(f^{\prime}, f, f^{\prime \prime}\right)$ of the homotopy cofibrations.

9. Triangulated categories: the stable homotopy category. Since $\mathfrak{e}^{\square}$ is provided with a suspension functor $\Sigma$, we associate with it, as in $\$ 1$, its stabilization $s e^{\square}$. In view of Theorem 7.1 and Corollary 7.2 this is a stable additive category. We shall show further, following Puppe [17], that $\mathfrak{S e}^{\square}$ is provided with a "triangulation." This is a notion due to Puppe; the terminology is however borrowed from Verdier.

We begin by recalling the definition of a triangulation. If $a$ is a stable additive category a triangulation of $a$ is a full replete subcategory $\Delta$ of $\operatorname{Tr} Q$ satisfying the following axioms:

$(\Delta 1)$ For all $A \in Q,\left(0 \rightarrow A_{\rightarrow}^{1} \rightarrow A \rightarrow 0\right) \in \Delta$.

$(\Delta 2)\left(A^{\prime a^{\prime \prime}} \rightarrow \stackrel{a^{\prime}}{\rightarrow} A^{\prime \prime} \stackrel{a}{\rightarrow} \Sigma A^{\prime}\right) \in \Delta$ if and only if $\left(A \stackrel{a^{\prime}}{\rightarrow} A^{\prime \prime} \stackrel{a}{\rightarrow} \Sigma A^{\prime-\Sigma a^{\prime \prime}} \rightarrow \Sigma A\right)$ $\in \Delta^{\prime}$.

$(\Delta 3)$ For any $a^{\prime}: A \rightarrow A^{\prime \prime}$ in $\propto$, there is a triangle $A^{\prime} \rightarrow A \stackrel{a^{\prime}}{\rightarrow} A^{\prime \prime} \rightarrow \Sigma A^{\prime}$ in $\Delta$.

$(\Delta 4)$ If

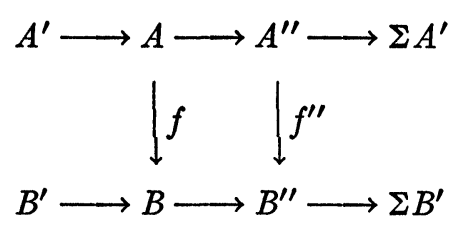

commutes and the rows are in $\Delta$, then there is an $f^{\prime}: A^{\prime} \rightarrow B^{\prime}$ such that $\left(f^{\prime}, f, f^{\prime \prime}\right)$ is a morphism in $\Delta$. 
Verdier adds an additional axiom, the so called "octahedral axiom," which we omit.

A category $Q$ which admits a triangulation is a triangulable category.

Proposition 9.1 (PUPPE). Let $\Delta$ be a triangulation of $a$. Then

(i) if $A^{\prime} \rightarrow A \rightarrow A^{\prime \prime} \rightarrow \Sigma A^{\prime}$ is in $\Delta$ the sequences

$\cdots \rightarrow \mathfrak{a}\left(-, A^{\prime}\right) \rightarrow \mathfrak{a}(-, A) \rightarrow \mathfrak{Q}\left(-, A^{\prime \prime}\right) \rightarrow \mathfrak{Q}\left(-, \Sigma A^{\prime}\right) \rightarrow \cdots$

$\cdots \leftarrow \mathfrak{a}\left(A^{\prime},-\right) \leftarrow \mathfrak{a}(A,-) \leftarrow \mathfrak{a}\left(A^{\prime \prime},-\right) \leftarrow \mathfrak{a}\left(\Sigma A^{\prime},-\right) \leftarrow \cdots$

are exact;

(ii) if $\left(f^{\prime}, f, f^{\prime \prime}\right)$ is a morphism in $\Delta$ and any two of $f^{\prime}, f, f^{\prime \prime}$ are isomorphisms, then so is the third;

(iii) if $A^{\prime} \rightarrow A \rightarrow A^{\prime \prime} \rightarrow \Sigma A^{\prime}, B^{\prime} \rightarrow B \rightarrow B^{\prime \prime} \rightarrow \Sigma B^{\prime}$ are in $\Delta$, then so is $A^{\prime} \oplus B^{\prime} \rightarrow A \oplus B \rightarrow A^{\prime \prime} \oplus B^{\prime \prime} \rightarrow \Sigma A^{\prime} \oplus \Sigma B^{\prime}$.

(iv) Moreover if $\Delta, \Delta^{\prime}$ are both triangulations of $A$ and $\Delta^{\prime} \subset \Delta$, then $\Delta^{\prime}=\Delta$.

For the proof, which is in any case quite easy, we refer to Puppe [17].

We may now introduce the cofibration-triangulation $\Delta_{\text {Cof }}$ of $\mathfrak{s e}^{\square}$. We recall that the functor $S: \mathfrak{e}^{\square} \rightarrow S \mathfrak{C}^{\square}$ is weakly stable, with an isomorphism $\sigma: S \Sigma \approx \Sigma S$. To each homotopy cofibration $X^{\prime} \stackrel{x^{\prime \prime}}{\rightarrow} X \stackrel{x^{\prime}}{\rightarrow} X^{\prime \prime}$ $\stackrel{x}{\rightarrow} \Sigma X^{\prime}$ in $\mathrm{e}^{\square}$ we associate the triangle $S X^{\prime S x^{\prime \prime}} \rightarrow S X \stackrel{S x^{\prime}}{\rightarrow} S X^{\prime \prime \sigma x^{\prime} S x} \underset{\rightarrow}{\rightarrow} S X^{\prime}$.

The full replete subcategory generated by the triangles $A^{\stackrel{a^{\prime \prime}}{\rightarrow}} A \stackrel{a^{\prime}}{\rightarrow} A^{\prime \prime}$ $\stackrel{a}{\rightarrow} \Sigma A^{\prime}$ such that for some even integer $h$

$$
\Sigma^{h} A^{\prime} \stackrel{\Sigma^{h} a^{\prime \prime}}{\longrightarrow} \Sigma^{h} A \stackrel{\Sigma^{h} a^{\prime}}{\longrightarrow} \Sigma^{h} A^{\prime \prime} \stackrel{\Sigma^{h} a}{\longrightarrow} \Sigma^{h+1} A^{\prime}
$$

is isomorphic to one of these is $\Delta_{\text {Cof }}$.

THEOREM 9.2. $\Delta_{\text {Cof }}$ is a triangulation of $\mathfrak{s e}^{\square}$.

Axiom $\Delta 1$ is trivially satisfied; $\Delta 2$ follows from Theorem $8.2 ; \Delta 3$ is a consequence of the mapping-cylinder axiom; and $\Delta 4$ follows from Lemma 8.3, after suspension.

10. The Freyd construction; universal homology of h-c-categories. It follows from Proposition 9.1(i) that in a triangulable category every morphism has a weak kernel and a weak cokernel. A result of Freyd [9] then asserts that each triangulable category has a universal full imbedding in an abelian category. For the sake of completeness we outline the relevant construction here.

If $Q$ is an additive category, we define $\Phi Q=Q \rightarrow / \mathscr{T}$ where $Q^{\rightarrow}$ is the category of morphisms in $Q$ and $\Re$ is the two-sided ideal consisting of morphisms $\left(f^{\prime}, f\right)$ in $\boldsymbol{Q}^{\rightarrow}$ : 


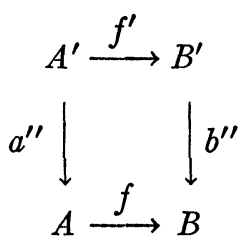

such that $f a^{\prime \prime}=0\left(=b^{\prime \prime} f^{\prime}\right)$. We regard $Q$ as a full subcategory of $\Phi Q$ via the imbedding $A \rightarrow 1_{A}$. An endofunctor $\Sigma$ of $a$ extends in the obvious way to $\Phi \propto$; if $Q$ is stable then so is $\Phi \propto$.

TheOREM 10.2 (FREYD). If $Q$ is triangulable, then $\Phi Q$ is abelian. Further $\Phi Q$ is Frobenius, i.e. there are both enough injectives and enough projectives, and these classes of objects coincide. The objects of a constitute both a resolving class of injectives and a resolving class of projectives in $\Phi \propto$. If a triangle in $a$ belongs to some triangulation, then it is an exact triangle in $\Phi \propto$. Finally, any stable functor $F: Q \rightarrow B$ into an abelian category $B$ which, for some triangulation $\Delta$, takes each triangle in $\Delta$ into an exact triangle of $B$, extends uniquely (up to isomorphism) to an exact functor $\Phi Q \rightarrow$ B.

We shall not give the proof (cf. [9, Theorem 3.1]), but the following observation may give an adequate idea of how it proceeds. Choose some triangulation $\Delta$ of $a$. Then the kernel, image, and cokernel of the morphism $\left(f^{\prime}, f\right)$ of $(10.1)$ are indicated in the diagram,

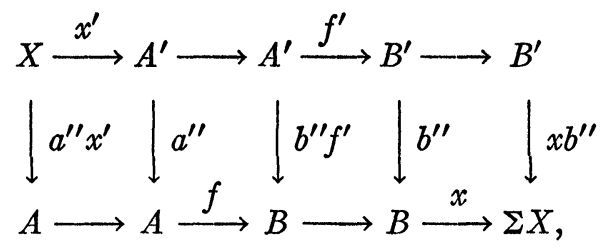

where $X \stackrel{x^{\prime}}{\rightarrow} A^{\prime b^{\prime \prime} f^{\prime}} \rightarrow B \Sigma \stackrel{x}{\rightarrow} X$ is in $\Delta$.

Now if $\mathfrak{C}$ is an h-c-category we write $H \mathcal{C}=\Phi S \mathfrak{C}^{\square}$ and let $h$ be the composition $\mathrm{C} \rightarrow \mathrm{e}^{\square S} \rightarrow \mathcal{S} \mathrm{e}^{\square} \rightarrow H \mathcal{C}$. We define $\partial: h T^{\prime \prime} \rightarrow \Sigma h T^{\prime}$ as follows. For any $X^{\prime} \rightarrow X \rightarrow X^{\prime \prime}$ in cof $\mathfrak{C}$ the connecting morphism $\delta$ gives a homotopy-cofibration $X^{\prime} \rightarrow X \rightarrow X^{\prime} \stackrel{\delta}{\rightarrow} \Sigma X^{\prime}$ in $\mathfrak{e}^{\square}$. Then, as in $\S 9$, $\sigma_{X}{ }^{\prime}(S \delta): S X^{\prime \prime} \rightarrow \Sigma S X^{\prime}$ in $S e^{\square}$. Thus $\partial=\sigma_{X}{ }^{\prime}(S \delta): h X^{\prime \prime} \rightarrow \Sigma h X^{\prime}$.

THEOREM $10.3(h, \partial)$ is the universal homology theory of $\mathfrak{C}$.

We observe first that $(h, \partial)$ is a homology theory. The homotopy axiom is trivially satisfied. The exactness axiom follows from Theorem 10.2 if we recall that for a cofibration $X^{\prime} \rightarrow X \rightarrow X^{\prime \prime}$ in $\mathfrak{e}$ the triangle $S X^{\prime} \rightarrow S X \rightarrow S X^{\prime \prime} \stackrel{\partial}{\rightarrow} \Sigma S X^{\prime}$ belongs to $\Delta_{\text {Cof }}$. 
But if $(h, \partial)$ is any cohomology theory from $\mathcal{C}$ to, say, $\mathcal{B}$, then $h$ factors as $\mathrm{e} \rightarrow \mathrm{e}^{\square} \stackrel{\bar{h}}{\rightarrow}$ B. But by Lemma $5.4, \bar{h}$ is weakly stable and thus, by Proposition 1.1, factors uniquely through astablefunctor $F: S \mathrm{e}^{\square} \rightarrow B$. But $F$ takes triangles in $\Delta_{\text {Cof }}$ into exact triangles in $B$ and, by Theorem 10.2, extends uniquely to a stable exact functor $H \mathfrak{e} \rightarrow$ .

\section{Chapter II}

11. Representability of group-valued homology theories. In view of Theorems 10.2, 10.3 we may use the techniques of homological algebra to obtain representability theorems for homology and cohomology theories with values in the category $a b^{\infty}$ of graded abelian groups, supplied with degree-shift as suspension functor. We confine ourselves here to a theorem asserting representability-inthe-limit. It is possible to obtain stronger results under suitable hypotheses involving completeness of the categories and continuity of the functors. But these matters do not seem to be well understood and we avoid them here.

We begin with a result in homological algebra which is undoubtedly well known. However, for lack of a suitable reference, we outline a proof here.

Proposition 11.1. If $a$ is a small abelian category, then any leftexact (=kernel-preserving) functor $F: Q^{\mathrm{op}} \rightarrow a b$ is a colimit of a directed system of representable functors:

$$
F=\operatorname{inj} \lim a\left(-, A_{\alpha}\right)
$$

where $\left\{A_{\alpha}, \phi_{\beta}^{\alpha}: A_{\alpha} \rightarrow A_{\beta}\right\}$ is a directed system in $Q$.

We recall that the category $\mathscr{L}$ of left-exact functors $\mathfrak{Q}^{\circ p} \rightarrow a b$ is abelian and that the representable functors $Q(-, A)$ form a set of small generators. Thus we have an exact sequence

$$
\Perp_{i} Q\left(-, A_{i}\right) \rightarrow \Perp_{j} Q\left(-, B_{j}\right) \rightarrow F \rightarrow 0
$$

in which the image of each $Q\left(-, A_{i}\right)$ lies in a finite coproduct of the $a\left(-, B_{j}\right)$. We may accordingly deduce from (11.2) the exact sequence

$$
\text { inj } \lim _{U, V} Q\left(-, \quad \Perp_{i \in U} A_{i}\right) \stackrel{\theta}{\rightarrow} \text { inj } \lim _{U, V} Q\left(-, \quad \Perp_{j \in V} B_{j}\right) \rightarrow F \rightarrow 0
$$

where $U, V$ run over pairs of finite sets of indices such that for $i \in U$, the image of $Q\left(-, A_{i}\right)$, is contained in $\Perp_{j \in V} Q\left(-, B_{j}\right)$. But

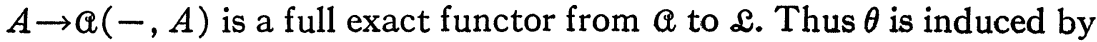


$\theta_{U V}: \Perp_{i \in U} A_{i} \rightarrow \Perp_{j \in V} B_{j}$. If the kernel of $\theta_{U V}$ is $C_{U V}$, we have, since inj $\lim$ is exact, $F \approx \operatorname{inj} \lim _{U, V} Q\left(-, C_{U V}\right)$.

We supplement this with the following observation.

Proposition 11.3. If $a$ is small abelian and $Q$ is a resolving set of injectives in $Q$, then any exact functor $F: Q^{\circ p} \rightarrow a b$ is isomorphic to $a$ colimit inj $\lim Q\left(-, X_{\alpha}\right)$ with $X_{\alpha} \in Q$.

By (11.2) we may assume $F=\operatorname{inj} \lim a\left(-, A_{\alpha}\right)$. For each $\alpha$ we choose a short exact sequence $0 \rightarrow A_{\alpha} \stackrel{\lambda_{\alpha}}{\rightarrow} X_{\alpha} \rightarrow B_{\alpha} \rightarrow 0$ with $X_{\alpha} \in Q$. Now for $\beta>\alpha$ consider the diagram

$$
\begin{aligned}
Q\left(A_{\alpha}, A_{\alpha}\right) & \ni 1_{\alpha} \\
\downarrow & \downarrow \\
Q\left(X_{\alpha}, A_{\beta}\right) \rightarrow Q\left(A_{\alpha}, A_{\beta}\right) & \ni \phi_{\beta}^{\alpha} .
\end{aligned}
$$

The limit of the second row, with respect to $\beta$, is $F X_{\alpha} \rightarrow F A_{\alpha}$, which is surjective. Thus for $\beta$ sufficiently large $\phi_{\beta}^{\alpha}$ is in the image of $Q\left(X_{\alpha}, A_{\beta}\right)$ $\rightarrow\left(A_{\alpha}, A_{\beta}\right)$, i.e. $\phi_{\beta}^{n}$ factors through $X_{\alpha}$.

Now let us choose for each $\alpha$ a sufficiently large $b(\alpha)>\alpha$ and a factorization $\phi_{b(\alpha)}^{\alpha}=\rho_{\alpha} \lambda_{\alpha}$. If we introduce in the index set the stronger ordering $\beta \geqq b(\alpha)$, then, by what we have just proved, the colimit does not change. But also the $X_{\alpha}$ form a directed system under the morphisms

$$
X_{\alpha} \stackrel{\rho_{\alpha}}{\rightarrow} A_{b(\alpha)} \stackrel{\stackrel{b(\alpha)}{\longrightarrow}}{\longrightarrow} A_{\beta} \stackrel{\lambda_{\beta}}{\rightarrow} X_{\beta}
$$

and, clearly, $F \approx$ inj $\lim a\left(-, X_{\alpha}\right)$.

The reader will readily supply the duals of these results as well as the following stable forms of them. If $a$ is a stable abelian category, we define for $A \in Q$ the stable representable functor $Q^{\infty}(-, A): Q^{\mathrm{op}} \rightarrow a b^{\infty}$ by $Q^{\infty}(X, A)_{q}=Q\left(X, \Sigma^{-q} A\right)$. If $Q$ is small stable abelian, then any stable left exact functor $Q^{o p} \rightarrow a b^{\infty}$ is a colimit of stable representable functors; a stable exact functor is a colimit of stable representable functors $Q^{\infty}\left(-, X_{\alpha}\right)$ with the $X_{\alpha}$ in some stable resolving class of injectives.

If we permit ourselves to define representable homology or cohomology theories on an h-c-category by using the obvious extension of the same terminology, then we have, as a corollary of the results above, the following theorem.

THEOREM 11.4. If $\mathfrak{e}$ is a small h-c-category, then any homology (cohomology) theory from $\mathrm{e}$ to $a b^{\infty}$ is a colimit of theories represented by an inverse (direct) system in $\mathrm{Se}^{\square}$. 
12. Rational homology on finite complexes; Dold's theorem. If $Q$ is an additive category and $\Lambda$ is a commutative ring, we define the category $\Lambda \otimes Q$ by giving it the same objects with the morphisms $(\Lambda \otimes Q)(A, B)=\Lambda \otimes \mathfrak{Q}(A, B)$, the composition being $\left(\lambda^{\prime} \otimes f^{\prime}\right)(\lambda \otimes f)$ $=\lambda^{\prime} \lambda \otimes f^{\prime} f$. Thus $f \rightarrow 1 \otimes f$ defines an additive functor $a \rightarrow \Lambda \otimes Q$.

$\Lambda \otimes Q$ is of course more than an additive category; it is (cf [8]) a category enriched over the closed category of $\Lambda$-modules or, as we shall say, a $\Lambda$-category. The notion of an enriched category is however sufficiently intuitive in these simple circumstances that we can appeal to intuition rather than general theory to pursue it.

If $\Lambda$ is a subring of $Q$, then $\Lambda \otimes Q$ has the following universal properties:

Proposition 12.1. If $F: Q \rightarrow B$ is an additive functor and $B$ is $a$ $\Lambda$-category, then $F$ may be factored as $Q \rightarrow \Lambda \otimes Q \stackrel{F^{\prime}}{\rightarrow} B$ where $F^{\prime}$ is a $\Lambda$ functor; $F^{\prime}$ is unique up to isomorphism.

Proposition 12.2. If $Q$ is abelian, then so is $\Lambda \otimes Q$. If $Q$ is an abelian $\Lambda$-category and the composition $Q \rightarrow \Lambda \otimes Q \stackrel{F^{\prime}}{\rightarrow} B$ is exact, where $F^{\prime}$ is a $\Lambda$-functor, then $F^{\prime}$ is exact.

The application we have in mind is to the category $J$ of finite $\mathrm{CW}$ complexes with basepoint made into an h-c-category as in (4.1). We denote by $\mathcal{V}$ the category of graded vector-spaces over $\boldsymbol{Q}$ which are finite dimensional in all degrees and nonzero in finitely many degrees. If $H$ is ordinary homology with coefficients $\boldsymbol{Q}$ (reduced homology, of course), then $H$ is a homology theory from $J$ to $V$ and thus factors as $J \rightarrow H J \stackrel{H^{\prime}}{\rightarrow} \cup$ with $H^{\prime}$ stable exact; and further, by Propositions $12.1,12.2, H^{\prime}$ factors as $H J \rightarrow Q \otimes H J^{H^{\prime \prime}} \rightarrow$ with $H^{\prime \prime}$ a stable exact Q-functor.

\section{LEMMA 12.3. $H^{\prime \prime}$ is an equivalence of Q-categories.}

This result belongs to the folklore. We shall not give a proof beyond observing that it follows easily by induction on, say, the number of cells involved from the fact that the stable homotopy groups of an $h$-sphere are finite in degrees different from $h$.

Now if we consider any homology theory on $J$ with values in a $Q$ category, we see that it must factor through $Q \otimes H J \approx \eta$. But a moment's reflection will show that there are very few $\boldsymbol{Q}$-functors on $v$. We have accordingly the following result.

THEOREM 12.4 (DoLD). If $h$ is any homology theory on $\mathrm{J}$ with values in a $\mathrm{Q}$-category, then $h \approx h S^{0} \otimes H$. 
Here $\otimes$ is the "symbolic" tensor product. Since the dual of a $Q$ category is once more a $\mathbf{Q}$-category, a similar result holds for cohomology.

13. Secondary composition. Toda (20) defined a "secondary composition," now generally called the "Toda bracket," in the category of topological spaces. Here we shall define a notion of secondary composition in an arbitrary stable additive category. If the category is triangulable, we shall exhibit a connection between the triangulations and the secondary compositions. In the succeeding paragraph we apply these observations to the original Toda bracket, which we define in any h-c-category.

If $Q$ is an additive category, we define the secondary domain and secondary codomain functors $D, D^{*}: a^{\rightarrow} \times a^{\rightarrow} \rightarrow a b$ as, respectively, the kernel and the cokernel in the exact sequences

$$
\begin{aligned}
& 0 \rightarrow D(f, g) \rightarrow a(Y, Z) \stackrel{\phi}{\rightarrow} a(X, Z) \oplus a(Y, W), \\
& a(X, Z) \oplus a(Y, W) \stackrel{\phi^{*}}{\rightarrow} a(X, W) \rightarrow D^{*}(f, g) \rightarrow 0
\end{aligned}
$$

where $f: X \rightarrow T, g: Z \rightarrow W, \phi u=(u f, g u)$ and $\phi^{*}(v, w)=g v-v f$. The images of $\phi, \phi^{*}$ are the coindeterminacy and indeterminacy of $(f, g)$.

If $Q$ is a stable additive category a secondary composition in $Q$ is a morphism $D \rightarrow D^{*}(\Sigma \times 1)$. The notation for such a morphism at $(f, g)$ is $u \mapsto[g, u, f]$. Since $D$ and $D^{*}$ are additive functors, $[g,-, f]$ is a homomorphism. The class of secondary compositions is of course a group.

If. $Q$ has a triangulation $\Delta$, we may associate to each sequence $X \stackrel{\leftrightarrow}{\rightarrow} Y \stackrel{u}{\rightarrow} Z \stackrel{g}{\rightarrow} W$ with $u f=0, g u=0$ a commutative diagram

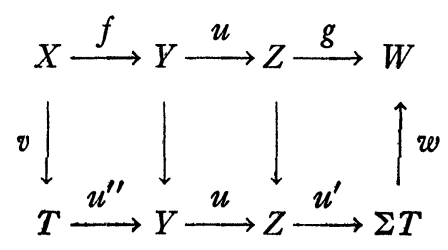

in which the second row belongs to $\Delta$. The morphisms $v$ and $w$ are not unique; $v$ has an indeterminacy of the form $\left(\Sigma^{-1} u^{\prime}\right) h, h: X \rightarrow \Sigma^{-1} Z$ and $w$ one of the form $k\left(\Sigma u^{\prime \prime}\right), k: \Sigma Y \rightarrow W$. Thus $w(\Sigma v) \in a(\Sigma X, W)$ is defined up to an indeterminacy of the form $w u^{\prime}(\Sigma h)+k \Sigma\left(u^{\prime \prime} v\right)$ $=g(\Sigma h)+k(\Sigma f)$. We denote by $[g, u, f]_{\Delta}$ the element of $D^{*}(f, g)$ represented by $w(\Sigma v)$. Naturality follows from standard arguments. Thus we have the following result. 
TheOREM 13.2. $\Delta \mapsto[-,-,-]_{\Delta}$ maps the class of triangulations of $a$ into the class of secondary compositions. Further, a triangle $A^{\prime} \stackrel{a^{\prime \prime}}{\rightarrow} A \stackrel{a^{\prime}}{\rightarrow} A^{\prime \prime} \stackrel{a}{\rightarrow} \Sigma A^{\prime}$ in a belongs to a triangulation $\Delta$ if and only if it is exact in $\Phi \propto$ and $\left[a, a^{\prime}, a^{\prime \prime}\right]_{\Delta} \ni 1_{\Sigma A^{\prime}}$. Thus $\Delta \mapsto[-,-,-]_{\Delta}$ is injective.

If the triangle belongs to $\Delta$, the conclusion is clear. Conversely, if $\left[a, a^{\prime}, a^{\prime \prime}\right]_{\Delta \ni} 1_{\Sigma A^{\prime}}$, then there is a diagram

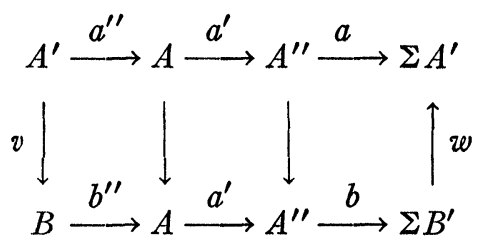

with the second row in $\Delta$ and $w(\Sigma v)=1_{\Sigma A^{\prime}}$. It follows from the 5lemma (in $\Phi Q$ ) that $\Sigma v, w$ are inverse isomorphisms, so that the two triangles are isomorphic.

14. The Toda bracket. Let $\mathfrak{e}$ be an h-c-category. If the sequence $X \stackrel{f}{\rightarrow} Y \stackrel{u}{\rightarrow} Z \stackrel{g}{\rightarrow} W$ satisfies $g u \simeq 0, u f \simeq 0$, then there is a commutative diagram

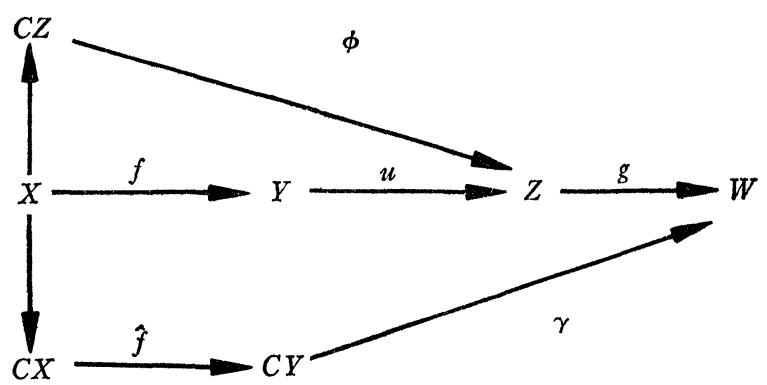

which determines a morphism $(g \phi \gamma \hat{f}): C X \cup C X \rightarrow W$. If we write $C X \stackrel{\xi}{\rightarrow} \Sigma X$ for the cofibre of $X \rightarrow C X$, then $(\xi 0): C X \cup C X \rightarrow \Sigma X$ is a homotopy equivalence; and we have, in $\mathfrak{e}^{\square}$, the composition $(g \phi \gamma \hat{f})(\xi 0)^{-1}: \Sigma X \rightarrow W$. This homotopy class depends on the choices of $\phi$ and $\gamma$ (though not on $\hat{f}$ ); we denote by $\{g, u, f\}$ the subset of $\mathrm{e}^{\square}(\Sigma X, W)$ so obtained. Following Toda [20] or Spanier [18] we have the following result.

LEMMA 14.1. $\{g, u, f\}$ depends only on the homotopy classes of $g, u, f$ and is a double coset of the subgroups $g \mathfrak{e}^{\square}(\Sigma X, Z), \mathrm{e}^{\square}(\Sigma Y, W)(\Sigma f)$. Further, $\Sigma\{g, u, f\} \subset-\{\Sigma g, \Sigma u, \Sigma f\}$.

Now in $\mathcal{S e}^{\square}$ suppose we have a sequence $\tilde{X} \stackrel{\tilde{f}}{\rightarrow} \tilde{Y} \stackrel{\tilde{u}}{\rightarrow} \tilde{Z} \stackrel{\tilde{g}}{\rightarrow} \tilde{W}$ with $\tilde{g} \tilde{u}=0, \tilde{u} \tilde{f}=0$. We define $[\tilde{g}, \tilde{u}, \tilde{f}]_{\text {Toda }} \in D^{*}(\Sigma \tilde{f}, \tilde{g})$ as follows. In $\mathcal{e}$ there 
is a sequence $X \stackrel{f}{\rightarrow} Y \stackrel{u}{\rightarrow} Z \stackrel{g}{\rightarrow} W$ as above such that $(\tilde{X} \rightarrow \tilde{Y} \rightarrow \tilde{Z} \rightarrow \tilde{W})$ $\approx \Sigma^{m}(S X \rightarrow S Y \rightarrow S Z \rightarrow S W)$. Then $[\tilde{g}, \tilde{u}, \tilde{f}]_{\text {Toda }} \supset(-1)^{m} \Sigma^{-m} S\{g, u, f\}$.

THEOREM 14.2. $[-,-,-]_{\mathrm{Toda}}$ is a secondary composition, and indeed $[-,-,-]_{\text {Toda }}=[-,-,-]_{\triangle_{\text {Cor }} \text {. }}$

The first assertion follows immediately from the lemma. The second follows from the first and the observation that if $A \stackrel{a}{\rightarrow} X \stackrel{b}{\rightarrow} X / A$ is a cofibration in $\mathbb{C}$ with connecting morphism $\delta \in \mathbb{C}^{\square}(X / A, \Sigma A)$, then $\{\delta, b, a\} \ni 1_{\Sigma A}$. But if $c: X / A \rightarrow \Sigma A$ represents $\delta$, then in the diagram

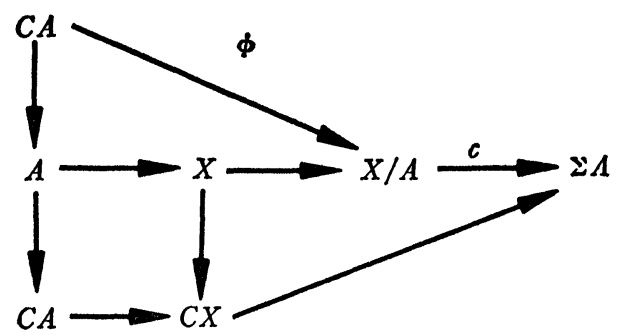

we may take $\phi=0$.

15. Injectives of $\Phi Q$. If $B$ is an abelian category, we write $I B$ for its full subcategory of injectives. If $B$ is stable, then so is $I B$. As we have seen (Theorem 10.2), if $Q$ is triangulable, then $a$ is a full subcategory of $I \Phi Q$; and indeed every injective of $\Phi Q$ is a retract of some $A \in Q$. It need not be the case that $A=I \Phi Q$; the following criterion, however, is more or less self-evident.

Propositron 15.1. If a is triangulable, then $A=I \Phi Q$ if and only if idempotents split in $Q$, i.e. if $f: A \rightarrow A, f^{2}=f$ implies

$$
f=\left(\begin{array}{ll}
1 & 0 \\
0 & 0
\end{array}\right)
$$

for some decomposition $A=A^{\prime} \oplus A^{\prime \prime}$.

The following result gives some insight into when this happens.

Proposition 15.2 (FREYd [10]). If $a$ is triangulable and has countable copowers, then idempotents split so that $Q=I \Phi Q$.

This implies, by a not-completely-trivial argument, the following.

Proposition 15.3 (Freyd [10]). If $J$ is the category of finite $C W$ complexes, then $S 5^{\square}=I H J$.

This serves as a model for other results of a similar character. The idea is to imbed in a larger category with copowers (in this case finite 
dimensional CW-complexes) and then show that the splitting in fact takes place in the original category.

We omit the proofs.

Our object here is to investigate the class $\Upsilon \propto$ of triangulations of a category $a$. We can do this only when $a=I \Phi Q$. Thus the following observations are relevant.

Lemma 15.4. If $\Delta$ is a triangulation of $Q$ and

$$
A^{\prime} \stackrel{\left(\begin{array}{c}
a^{\prime \prime} \\
0
\end{array}\right)}{\longrightarrow} A \oplus X \stackrel{a^{\prime} \oplus 1}{\longrightarrow} A^{\prime \prime} \oplus X \stackrel{(a 0)}{\longrightarrow} \Sigma A^{\prime}
$$

is in $\Delta$, then so is $A^{\prime a^{\prime \prime}} \rightarrow A \stackrel{a^{\prime}}{\rightarrow} A^{\prime \prime} \stackrel{a}{\rightarrow} \Sigma A^{\prime}$.

For there is a triangle $B^{\prime} \rightarrow A \stackrel{a^{\prime \prime}}{\rightarrow} A^{\prime \prime} \rightarrow \Sigma B^{\prime}$ in $\Delta$ and thus a commutative diagram

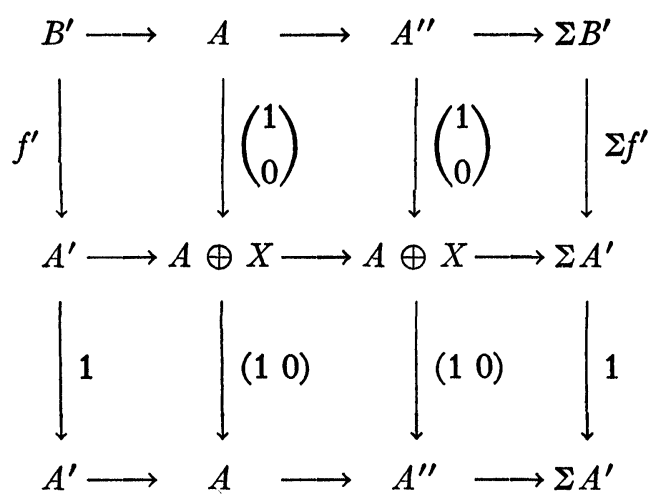

Since the last row is exact in $\Phi Q$ the 5-lemma implies that it is isomorphic to the first one.

If we define a split triangle as one of the form

$$
X^{\prime} \oplus \Sigma^{-1} X^{\prime \prime} \stackrel{1 \oplus 0}{\longrightarrow} X^{\prime} \oplus X \stackrel{0 \oplus 1}{\longrightarrow} X^{\prime \prime} \oplus X \stackrel{1 \oplus 0}{\longrightarrow} X^{\prime \prime} \oplus \Sigma X^{\prime},
$$

it is clear that the split triangles belong to every triangulation.

PRoposition 15.5. If $a$ is a triangulable category, then every triangulation $\Delta$ of $Q$ is contained in a unique triangulation $\Delta^{\prime}$ of $I \Phi Q$; and $a$ triangle $\boldsymbol{A}$ of $I \Phi Q$ is in $\Delta^{\prime}$ if and only if for some split triangle $X$, $\boldsymbol{A} \oplus \boldsymbol{X} \in \Delta$.

If we show that the $\Delta^{\prime}$ so defined is a triangulation of $I \Phi Q$, then, since $\Delta^{\prime} \cap \operatorname{Tr} a$ is obviously a triangulation of $a$, we have by (Proposi- 
tion 9.1(iv)) $\Delta=\Delta^{\prime} \cap \operatorname{Tr}$ Q. The uniqueness of $\Delta^{\prime}$ follows from (Proposition 9.1(iii), (iv)).

Now $\Delta^{\prime}$ clearly satisfies axioms $\Delta 1,2$. If $A \stackrel{a^{\prime}}{\rightarrow} A^{\prime \prime}$ in $I \Phi Q$, then for suitable $X, X^{\prime \prime}$ there is a triangle $W \rightarrow A \oplus X^{a^{\prime} \oplus 0} \rightarrow A^{\prime \prime} \oplus X^{\prime \prime} \rightarrow \Sigma W$ in $\Delta$. But $X$ is projective and $X^{\prime \prime}$ is injective. Thus the split triangle $\Sigma^{-1} X^{\prime \prime} \oplus X \rightarrow X \rightarrow X^{\prime \prime} \rightarrow X^{\prime \prime} \oplus \Sigma X$ can be split off, leaving a triangle $A^{\prime} \rightarrow A \stackrel{a^{\prime}}{\rightarrow} A^{\prime \prime} \rightarrow \Sigma A^{\prime}$ in $\Delta^{\prime}$.

Finally, if $A \oplus X, B \oplus Y$ are in $\Delta$, where $X, Y$ are split triangles, and $f: A \rightarrow B, f^{\prime \prime}: A^{\prime \prime} \rightarrow B^{\prime \prime}$ form a commuting square in $\Phi Q$, then there is a $g^{\prime}: A^{\prime} \oplus X^{\prime} \rightarrow B^{\prime} \oplus Y^{\prime}$ such that $\left(g^{\prime}, f \oplus 0, f^{\prime \prime} \oplus 0\right)$ is a morphism in $\Delta$. But then

$$
\left(\begin{array}{ll}
(1 & 0) g^{\prime}
\end{array}\left(\begin{array}{l}
1 \\
0
\end{array}\right), f, f^{\prime \prime}\right)
$$

is a morphism in $\Delta^{\prime}$.

In particular, if $Q$ is triangulable then so is $I \Phi Q$. It seems appropriate to extend the use of the term triangulable to stable Frobenuis categories $B$ for which $I B$ is triangulable in the primary sense; clearly for such categories $\Phi I ß \approx \beta$. In any case, for any triangulable $Q$,

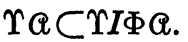

Similarly, we shall speak of triangulations of $B$ (i.e. of $I B$ ) and write $\Upsilon \otimes$ for $\Upsilon I B$.

16. The class of triangulations. We shall now compute the class of triangulations of a stable Frobenius category B. We make the preliminary observation that these classes are the same for equivalent categories. If $F: Q^{\prime} \rightarrow Q$ is a stable equivalence of stable additive categories then $\Delta \mapsto F^{-1}(\Delta)$ gives a bijection $\Upsilon Q \approx \Upsilon Q^{\prime}$.

Now $B$, being a Frobenius category, may be regarded as an h-ccategory as in (4.4), and, since injectives and projectives coincide, the suspension functor in $B^{\square}$, which we shall denote by $S$ to distinguish it from the suspension $\Sigma$ of $B$, is an equivalence. Since we may replace $B$ by an equivalent category we may suppose without loss of generality that it is possible to choose cones $C_{A}: A \rightarrow C A$ in such a way that

$$
\begin{aligned}
\otimes^{\square} & =S B^{\square}, \\
C_{\Sigma A} & =\Sigma C_{A}: \Sigma A \rightarrow C \Sigma A=\Sigma C A .
\end{aligned}
$$

Now $\Sigma$ leaves $I \leftrightarrow$ invariant and thus defines an automorphism, which we again denote by $\Sigma$, of $B^{\square}$. It follows at once from (16.1) that 


$$
S \Sigma=\Sigma S \quad \text { on } Q^{\square} \text {. }
$$

Both $S$ and $\Sigma$ extend canonically to $\Phi B^{\square}=H \otimes$ and the commutativity continues of course to hold there.

Since we have chosen cones in $B$ we have for each $A \in B$ a standard injective resolution $\hat{A}=\left(C A \rightarrow C S A \rightarrow C S^{2} A \rightarrow \cdots\right)$, with $Z^{q} \hat{A}=S^{q} A$. Suppose also that $A$ occurs as the image of $X^{\prime \prime}$ in the exact triangle of injectives

$$
X=\left(X^{\prime} \stackrel{x^{\prime \prime}}{\longrightarrow} X \stackrel{x^{\prime}}{\longrightarrow} X^{\prime \prime} \stackrel{x}{\longrightarrow} \Sigma X^{\prime}\right) .
$$

Then we may construct another injective resolution

$$
\hat{X}=\left(X \stackrel{x^{\prime}}{\rightarrow} X^{\prime \prime} \stackrel{x}{\rightarrow} \Sigma X^{\prime} \stackrel{-\Sigma x^{\prime \prime}}{\longrightarrow} \Sigma X \stackrel{-\Sigma x^{\prime}}{\longrightarrow} \Sigma X^{\prime \prime} \rightarrow \cdots\right) .
$$

Here $Z^{3} \hat{X}=\Sigma A$. By the usual comparison theorem of homological algebra there is a unique chain-homotopy class of morphisms $\iota_{A}: \hat{X} \rightarrow \hat{A}$ over $1_{A}$, and $Z^{3} \iota_{A}$ determines a unique injective-homotopy-class $\delta_{X} \in B^{\square}\left(\Sigma A, S^{3} A\right)$ which is in fact an isomorphism in $B^{\square}$.

Now suppose $\Delta$ is a triangulation of $B$. Then every $A$ appears as the image of $x^{\prime \prime}$ for some $X \in \Delta$. If it is also, similarly, the image of $w^{\prime \prime}$ in a triangle $\mathrm{W} \in \Delta$, then there is a morphism $X \rightarrow \mathrm{W}$ of triangles which gives the identity on $A$ and thus also on $\Sigma A$. It follows that $\delta_{W}=\delta_{X}$, and we have accordingly a morphism $\delta(\Delta)_{A}: \Sigma A \rightarrow S^{3} A$ in $B^{\square}$. From the comparison theorem, once more we see easily that $\delta(\Delta)$ is natural, i.e. $\delta(\Delta): \Sigma \approx S^{3}$.

LEMMA 16.3. For any triangulation $\Delta$ of $B, \delta(\Delta) S+S \delta(\Delta)=0$.

We may, for any $A \in \Theta$, choose a triangle $X$ as above with $A$ the image of $x^{\prime \prime}$ and $A \rightarrow X$ the cone $A \rightarrow C A$. We then have a commutative diagram

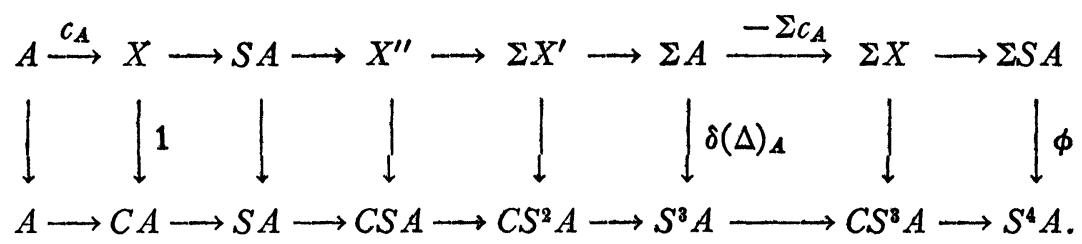

By axiom $\Delta 2$ we may identify $\phi$ as $\delta(\Delta)_{S A}$. But since $\Sigma c_{\Lambda}=c_{\Sigma A}$ it is also $-S \delta(\Delta)_{A}$.

THEOREM 16.4. The operation $\delta$ gives a bijective correspondence between triangulations of $B$ and isomorphisms $\Sigma \rightarrow S^{3}$ on $B$ which anticommute with $S$. 
It will be sufficient to prove the following assertion: if $\theta: \Sigma \approx S^{3}$ anticommutes with $S$, then the class $\Delta$ of exact triangles

$$
X=\left(X^{\prime} \stackrel{x^{\prime \prime}}{\longrightarrow} X \stackrel{x^{\prime}}{\longrightarrow} X^{\prime \prime} \stackrel{x}{\longrightarrow} \Sigma X^{\prime}\right)
$$

of injectives such that $\delta_{X^{\prime}}=\theta_{\operatorname{im} x^{\prime \prime}}$ is a triangulation of $B$.

Axiom $\Delta 1$ is trivially satisfied; axiom $\Delta 2$ follows by reversing the argument of Lemma 16.3. For $\Delta 3$, suppose $X^{\prime} \stackrel{x^{\prime \prime}}{\rightarrow} X$ and let $B^{\prime} \rightarrow X^{\prime}$ $\rightarrow A \rightarrow X \rightarrow B$ indicate the kernel, image, and cokernel. Then we have in $B$ commutative diagrams

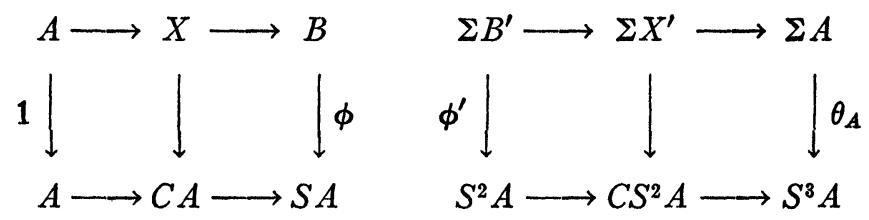

since $C A$ is injective and $\Sigma X^{\prime}$ is projective. Moreover $\phi$ and $\phi^{\prime}$ are isomorphisms in $B^{\square}$. Thus there is an exact sequence $0 \rightarrow B \rightarrow X^{\prime \prime}$ $\rightarrow \Sigma B^{\prime} \rightarrow 0$ with a morphism $\left(\phi, \psi, \phi^{\prime}\right)$ in to $0 \rightarrow S A \rightarrow C S A \rightarrow S^{2} A \rightarrow 0$. Since $\phi, \phi^{\prime}$ are isomorphisms modulo injectives, so also is $\psi$, i.e. $X^{\prime \prime}$ is injective. But then the triangle $X^{\prime} \rightarrow X \rightarrow X^{\prime \prime} \rightarrow \Sigma X^{\prime}$ is in $\Delta$.

Finally, suppose that $X$ and $Y$ are in $\Delta$, that $f: X^{\prime} \rightarrow Y^{\prime}, f: X \rightarrow Y$, and that $f x^{\prime \prime}=y^{\prime \prime} f^{\prime}$. If we write $A, B$ for the images of $x^{\prime \prime}, y^{\prime \prime}$, there is certainly a commutative diagram

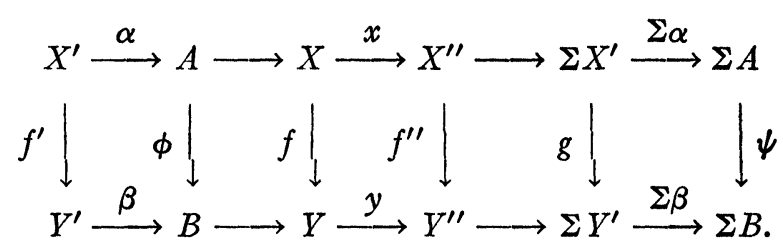

But $\delta_{Y} \psi=\left(S^{3} \phi\right) \delta_{X}$ in $B^{\square}$ because of the naturality of $\theta$ and the fact that, for $\hat{\phi}: \hat{A} \rightarrow \hat{B}$ covering $\phi, Z^{3} \hat{\phi}=S^{3} \phi$. Thus $\psi \simeq \Sigma \phi$; we may in fact suppose $\psi=\Sigma \phi$. But $(\Sigma \beta)\left(g-\Sigma f^{\prime}\right)=\Sigma\left(\phi \alpha-\beta f^{\prime}\right)=0$ so that $\Sigma f^{\prime}=g+y h$ for some $h: \Sigma X^{\prime} \rightarrow Y^{\prime \prime}, \Sigma X^{\prime}$ being projective as well as injective. But then $\left(f^{\prime}, f, f^{\prime \prime}+h x\right)$ is a morphism of triangles.

If $B$ is triangulable, then the group consisting of those automorphisms of $\Sigma$ which commute with $S$ operates freely and transitively, by composition, on the set of isomorphisms $\Sigma \approx S^{3}$ which anticommute with $S$ and thus, by the theorem just proved, on $\Upsilon \leftrightarrow$. Since $\Sigma$ is an automorphism of $\beta^{\square}$ which commutes with $S$ we deduce immediately the following result. 
COROLLARY 16.5. The group consisting of those automorphisms of the identity functor of $H B$ which commute with $S$ operates freely and transitively on $\Upsilon B$.

17. An example: triangulations of $\mathrm{HT}$. We shall not compute the class $\Upsilon H J$ of triangulations of $H J$ (which by Proposition 15.3 coincides with $\Upsilon S J^{\square}$ ) but shall content ourselves with showing that there are uncountably many of them. By Corollary 16.5 this can be done by showing that the group of those automorphisms of $H^{2} J=H H J$ which commute with the suspension $S$ has an uncountable subgroup.

An additive category $Q$ is a torsion category if each object $A$ is a torsion object, i.e. one such that for some integer $h, h \cdot 1_{A}=0$.

LeMma 17.1. $H^{2} 3$ is a torsion category.

It is of course sufficient to show that $(H J)^{\square}$ is a torsion category. Since $\Sigma$ is an automorphism of $H J$ it is in fact sufficient to show that for any morphism $f: X \rightarrow Y$ in $\Im$ the corresponding object of $(H J)^{\square}$ is a torsion object, i.e. that for some $h$ the morphism

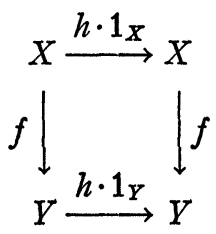

factors through an injective. As in $\$ 12$, let $H$ denote ordinary homology with rational coefficients. Then it follows from Lemma 12.3 that

(i) If $w: X \rightarrow Y$ in $s 5^{\square}$ and $H w=0$, then, for some $n=1,2, \cdots$, $n w=0$.

(ii) There is a $g: Y \rightarrow X$ and an $m=1,2, \cdots$ such that $H(f g f)$ $=m H f$

But this implies that for a suitable positive integer $n$ the morphism $(n g f, n f g)$ is the same as $\left(m n \cdot 1_{X}, m n \cdot 1_{Y}\right)$ and thus has the factorization

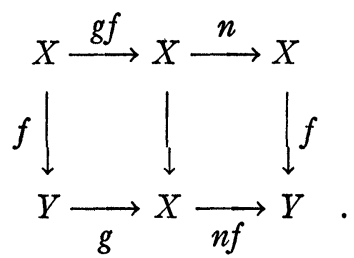

Now any torsion category $a$ in which idempotents split is the coproduct of its $p$-primary subcategories, i.e. the full subcategories $a_{p}$ of objects annihilated by some power of the prime $p$. In particular 
$H^{2} J=\Pi_{p}\left(H^{2} J\right)_{p}$. Such $p$-primary subcategories are of course stable under automorphisms of the category. Thus the group $G$ of those automorphisms of the identity functor of $H^{2} J$ which commute with $S$ is $\Pi_{p} G_{p}$, where $G_{p}$ is the corresponding group for $\left(H^{2} J\right)_{p}$.

But in a $p$-primary torsion category multiplication by any unit of the ring $\boldsymbol{Z}_{p}{ }^{*}$ of $p$-adic integers is clearly an automorphism of the identity functor and certainly commutes with any additive endofunctor. This defines a homomorphism from the group $u Z_{p}^{*}$ of such units into $G_{p}$. The example of the Moore-spaces corresponding to the groups $\boldsymbol{Z} / p^{k} \boldsymbol{Z}$ shows that this is a monomorphism.

THEOREM 17.2. $\Pi_{p} u\left(Z_{p}^{*}\right)$ operates freely on $\Upsilon \boldsymbol{\Upsilon} H$ J.

Chapter III

18. Cofibrations in Cof $\mathfrak{C}$. If $(\mathfrak{e}, \operatorname{Cof} \mathfrak{C})$ is a c-category, so that Cof $\mathfrak{C}$ is a system of cofibrations in $\mathfrak{C}$, i.e. a full subcategory of $\mathfrak{C}^{\rightarrow}$ satisfying axioms (C0), (C1) of $\$ 3$, we define a full subcategory $\mathrm{Cof}^{2} \mathfrak{C} \subset(\text { Cof } \mathfrak{e})^{\rightarrow}$ as follows. Suppose $\left(f^{\prime}, f\right)$ is a morphism in Cof $\mathfrak{C}$,

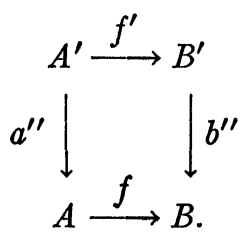

Then the pushout $A \cup B^{\prime}$ exists, and $\left(f b^{\prime \prime}\right): A \cup B^{\prime} \rightarrow B$. Cof ${ }^{2} \mathcal{e}$ contains as objects those $\left(f^{\prime}, f\right)$ for which $f^{\prime}$ and $\left(f b^{\prime \prime}\right)$ are in Cof $\mathbb{C}$. It follows of course that $f$ is also in Cof $\mathfrak{e}$ if $\left(f^{\prime}, f\right)$ is in $\operatorname{Cof}^{2} \mathfrak{e}$.

Proposition 18.1. Cof $^{2} \mathfrak{e}$ is a system of cofibrations in Cof $\mathfrak{e}$.

For axiom ( $\mathrm{C} 0)$ we need only check closure under composition. Suppose also $\left(g^{\prime}, g\right) \in \operatorname{Cof}^{2} \mathcal{C}$, so that we have a commutative diagram

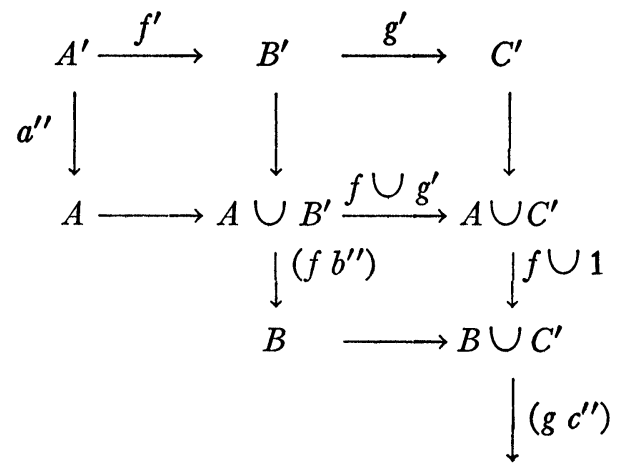

C. 
Here $f \cup 1$ is the pushout of $\left(f b^{\prime \prime}\right)$ and is thus a cofibration. But $\left(g c^{\prime \prime}\right)(f \cup 1)=\left(g f c^{\prime \prime}\right): A \cup C^{\prime} \rightarrow C$ so that $\left(g^{\prime} f^{\prime}, g f\right) \in \operatorname{Cof}^{2} \mathcal{e}$.

To verify (C1) suppose as above that $\left(f^{\prime}, f\right) \in \operatorname{Cof}^{2} \mathfrak{e}$ and that $\left(g^{\prime}, g\right): a^{\prime \prime} \rightarrow c^{\prime \prime}$ is any morphism. Then we may construct the pushouts $C^{\prime} \cup B^{\prime}, C \cup B$, and it is easy to see that the diagram

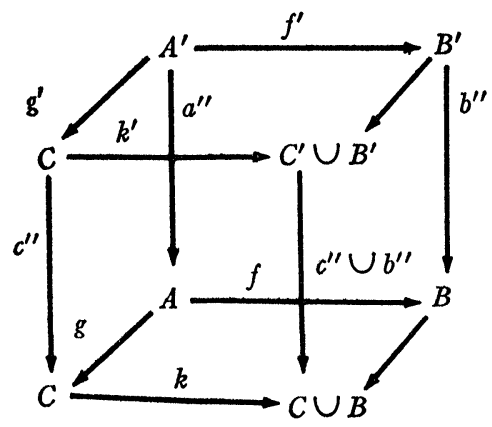

regarded as a square in $\mathfrak{e}^{\rightarrow}$ is a pushout. We must show that $c^{\prime \prime} \cup b^{\prime \prime}$ is a cofibration and that $\left(k^{\prime}, k\right) \in \operatorname{Cof}^{2} \mathbf{e}$.

But it is easy to see that, as pushouts, $C \cup\left(C^{\prime} \cup B^{\prime}\right)=C \cup B^{\prime}$. Mak-

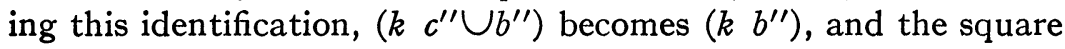

$$
\begin{aligned}
& A \cup B^{\prime} \stackrel{\left(f b^{\prime \prime}\right)}{\longrightarrow} B \\
& \quad \downarrow f \cup 1 \\
& C \cup B^{\prime} \stackrel{\left(k b^{\prime \prime}\right)}{\longrightarrow} C \cup B
\end{aligned}
$$

is a pushout. Since $C^{\prime} \cup B^{\prime c^{\prime \prime} \cup 1} \longrightarrow C \cup B^{\prime}$ is itself a pushout of $c^{\prime \prime},\left(\begin{array}{ll}k & b^{\prime \prime}\end{array}\right)$ $\cdot\left(c^{\prime \prime} \cup 1\right)=c^{\prime \prime} \cup b^{\prime \prime} \in$ Cof $\mathcal{C}$, and the conclusion follows.

We may now iterate this construction: if $(\mathfrak{e}$, Cof $\mathfrak{e})$ is a c-category, we set $\operatorname{Cof}^{0} \mathfrak{C}=\mathfrak{e}, \operatorname{Cof}^{n+1} \mathfrak{C}=\operatorname{Cof}\left(\operatorname{Cof}^{n} \mathfrak{C}\right)$. Then $\left(\operatorname{Cof}^{n} \mathfrak{e}, \operatorname{Cof}^{n+1} \mathfrak{e}\right)$ is a c-category for $n=0,1, \cdots$.

19. Homotopies in Cof $\mathfrak{e}$. We suppose now that (e, Cof $\mathfrak{e}, \simeq$ ) is an h-c-category. We shall define a homotopy relation in Cof $\mathfrak{e}$ by introducing the notion of a cylinder over an object of Cof $\mathfrak{e}$; in other words we shall copy the "topological" definition of the homotopy relation (compare $\$ 6$ ).

If $a^{\prime \prime}: A^{\prime} \rightarrow A$ is an object of Cof $\mathcal{C}$, a cylinder over $a^{\prime \prime}$ is a cofibration $\left(\xi^{\prime}, \xi\right): a^{\prime \prime} \bigvee a^{\prime \prime} \rightarrow x^{\prime \prime}$ in Cof $\mathcal{e}$ such that there exists a morphism $\left(\hat{\xi}^{\prime}, \hat{\xi}\right): x^{\prime \prime} \rightarrow a^{\prime \prime}$ with the properties that (i) both $\hat{\xi}^{\prime}$ and $\hat{\xi}$ are homotopy equivalences, (ii) $\left(\hat{\xi}^{\prime}, \hat{\xi}\right)\left(\xi^{\prime}, \xi\right)=\left(1_{a^{\prime \prime}} 1_{a^{\prime \prime}}\right)$. Such a morphism is a projection of the cylinder. 
The following lemmas are needed in order to define the homotopy relation.

Lemma 19.1. If $\left(f^{\prime}, f\right): a^{\prime \prime} \rightarrow b^{\prime \prime}$ is a morphism in Cof $\mathfrak{C}$, then it has a factorization

$$
a^{\prime \prime} \stackrel{\left(\alpha^{\prime}, \alpha\right)}{\longrightarrow} x^{\prime \prime} \stackrel{\left(\beta^{\prime}, \beta\right)}{\longrightarrow} b^{\prime \prime}
$$

such that $\left(\alpha^{\prime}, \alpha\right)$ is a cofibration in $\operatorname{Cof} \mathrm{e}$ and both $\beta^{\prime}$ and $\beta$ are homotopy equivalences.

Let $A^{\prime} \stackrel{\alpha^{\prime}}{\rightarrow} X^{\prime} \stackrel{\beta^{\prime}}{\rightarrow} B^{\prime}$ be a mapping cylinder for $f^{\prime}$. Then the pushout $A \cup X^{\prime}$ is mapped by $\left(f b^{\prime \prime} \beta^{\prime}\right)$ to $B$. This in turn has a mapping cylinder $A \cup X^{\prime} \rightarrow X \stackrel{\beta^{\prime}}{\rightarrow} B$. We need only let $x^{\prime \prime}, \alpha$ be, respectively, the compositions $X^{\prime} \rightarrow A \cup X^{\prime} \rightarrow X$ and $A \rightarrow A \cup X^{\prime} \rightarrow X$.

Corollary 19.2. Every $a^{\prime \prime} \in$ Cof e has a cylinder.

Lemma 19.3. If $\left(f^{\prime}, f\right): a^{\prime \prime} \rightarrow b^{\prime \prime}$ is a cofibration in Cof $e$ and both $f^{\prime}$ and $f$ are homotopy equivalences, then $\left(f^{\prime}, f\right)$ has a retraction, i.e. a leftinverse $\left(r^{\prime}, r\right): b^{\prime \prime} \rightarrow a^{\prime \prime}$.

In the diagram

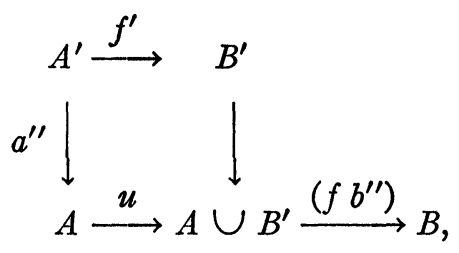

$u$, as a pushout of $f^{\prime}$, is a homotopy equivalence. Since $\left(f b^{\prime \prime}\right) u=f$, $\left(f b^{\prime \prime}\right)$ is also a homotopy equivalence. Thus $f^{\prime}$ and $\left(f b^{\prime \prime}\right)$, being cofibrations in $\mathcal{C}$, have retractions $r^{\prime}, s$. Then $r=\left(1 f^{\prime}\right) s$ gives the retraction $\left(r^{\prime}, r\right)$ of $\left(f^{\prime}, f\right)$.

LEMmA 19.4. If $\left(\xi^{\prime}, \xi\right): a^{\prime \prime} \vee a^{\prime \prime} \rightarrow x^{\prime \prime}$, and $\left(\eta^{\prime}, \eta\right): b^{\prime \prime} \vee b^{\prime \prime} \rightarrow y^{\prime \prime}$ are cylinders, and $\left(f^{\prime}, f\right): a^{\prime \prime} \rightarrow b^{\prime \prime}$ is any morphism, then there is a $\left(g^{\prime}, g\right): x^{\prime \prime} \rightarrow y^{\prime \prime}$ such that $\left(g^{\prime} \xi^{\prime}, g \xi\right)=\left(\eta^{\prime} f^{\prime}, \eta f\right)$.

Let us choose first projections $\left(\hat{\xi}^{\prime}, \hat{\xi}\right),\left(\hat{\eta}^{\prime}, \hat{\eta}\right)$ for the two cylinders. Now notice that $x^{\prime \prime} \cup y^{\prime \prime}: X^{\prime} \cup Y^{\prime} \rightarrow X \cup Y$ is a pushout in Cof $\mathfrak{e}$, so that all the morphisms in

$$
\begin{aligned}
a^{\prime \prime} \vee a^{\prime \prime} \stackrel{\left(\xi^{\prime}, \xi\right)}{\longrightarrow} x^{\prime \prime} & \mid \\
y^{\prime \prime} & \left.\left.\downarrow f^{\prime} \vee f^{\prime}\right), \eta(f \vee f)\right) \\
&
\end{aligned}
$$


are in Cof $^{2}$ e. Using Lemma 19.1 we construct a factorization

$$
x^{\prime \prime} \cup y^{\prime \prime} \rightarrow z^{\prime \prime} \stackrel{\left(\xi^{\prime}, \hat{\xi}\right)}{\longrightarrow} b^{\prime \prime}
$$

of $\left(\left(f^{\prime} \hat{\xi}^{\prime}, f \xi\right) \cdot\left(\hat{\eta}^{\prime}, \hat{\eta}\right)\right)$. Let us write $\left(\rho^{\prime}, \rho\right): y^{\prime \prime} \rightarrow z^{\prime \prime}$ for the composition $y^{\prime \prime} \rightarrow x^{\prime \prime} \cup y^{\prime \prime} \rightarrow z^{\prime \prime}$. Since we have a commutative diagram

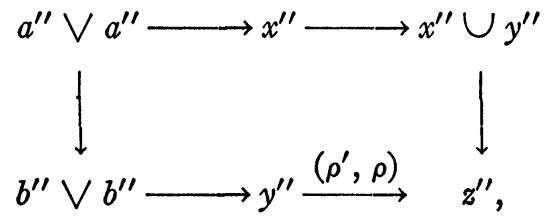

it will be sufficient to show that $\left(\rho^{\prime}, \rho\right)$ has a left inverse $\left(\lambda^{\prime}, \lambda\right)$.

But $\left(\rho^{\prime}, \rho\right)$ is in $\operatorname{Cof}^{2} \mathcal{C}$ and both $\rho^{\prime}$ and $\rho$ are homotopy equivalences. Thus the conclusion follows from Lemma 19.3.

LemmA 19.5. If $\left(\left(\xi_{0}^{\prime} \xi_{1}^{\prime}\right),\left(\xi_{0} \xi_{1}\right)\right): a^{\prime \prime} \bigvee a^{\prime \prime} \rightarrow x^{\prime \prime}$ is a cylinder over $a^{\prime \prime}$, then so are $\left(\left(\xi_{1}^{\prime} \xi_{0}^{\prime}\right),\left(\xi_{1} \xi_{0}\right)\right)$ and $\left(\left(\xi_{0}^{\prime} \xi_{2}^{\prime}\right),\left(\xi_{0} \xi_{2}\right)\right): a^{\prime \prime} \vee a^{\prime \prime} \rightarrow x^{\prime \prime} \oplus x^{\prime \prime}$, where $x^{\prime \prime} \oplus x^{\prime \prime}: X^{\prime} \oplus X^{\prime} \rightarrow X \oplus X$ is defined as in $\$ 6$.

We may now, finally, define the homotopy relation $\simeq_{1}$ in Cof $\mathrm{e}$. If $\left(f_{0}^{\prime}, f_{0}\right),\left(f_{1}^{\prime}, f_{1}\right): a^{\prime \prime} \rightarrow b^{\prime \prime}$, then $\left(f_{0}^{\prime}, f_{0}\right) \simeq_{1}\left(f_{1}^{\prime}, f_{1}\right)$ if for any cylinder $\left(\xi^{\prime}, \xi\right): a^{\prime \prime} \vee a^{\prime \prime} \rightarrow x^{\prime \prime}$ there exists a $\left(g^{\prime}, g\right): x^{\prime \prime} \rightarrow b^{\prime \prime}$ such that $\left(g^{\prime} \xi^{\prime}, g \xi\right)$ $=\left(\left(f_{0}^{\prime} f_{1}^{\prime}\right),\left(f_{0} f_{1}\right)\right)$.

PROPOSITION 19.6. $\simeq_{1}$ is a congruence in Cof $\mathfrak{e}$.

It follows immediately from Lemma 19.4 that if $\left(g^{\prime}, g\right)$ exists for one cylinder, then it exists for all. Thus Lemma 19.5 implies that $\simeq_{1}$ is an equivalence relation. Finally, Lemma 19.4 again shows that it is indeed a congruence.

20. Cof $\mathfrak{C}$ as an h-c-category. We shall prove the following statement.

Theorem 20.1. If (e, Cof $\mathfrak{e}, \simeq$ ) is an h-c-category, then so also is (Cof $\mathcal{e}, \operatorname{Cof}^{2} \mathcal{e}, \simeq_{1}$ ).

The proof will be preceded by several lemmas.

LEMMA 20.2. If the diagram

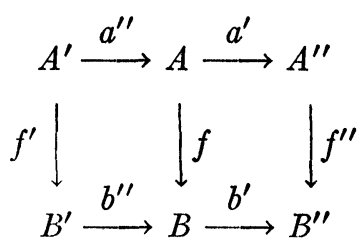


is a cofibration in Cof $\mathcal{e}$ (together with its cofibre) and $f^{\prime}, f$ are homotopy equivalences, then $f^{\prime \prime}$ is also a homotopy equivalence.

The morphism $A \rightarrow B^{\prime} \cup A$, as the pushout of $f^{\prime}$, is a homotopy equivalence. Since $f$ is the composition $A \rightarrow B^{\prime} \cup A \stackrel{\left(b^{\prime \prime} f\right)}{\rightarrow} B,\left(b^{\prime \prime} f\right)$ is also a homotopy equivalence. But

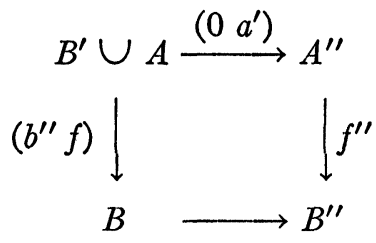

is easily seen to be a pushout. Since $\left(a^{\prime \prime}, b^{\prime \prime}\right)$ is a cofibration in Cof $\mathfrak{C}$, $\left(b^{\prime \prime} f\right)$ is a cofibration and the conclusion follows.

Lemma 20.3. If $\left(f^{\prime}, f\right): a^{\prime \prime} \rightarrow b^{\prime \prime}$ is a cofibration in Cof $\mathfrak{C}$, then there is a commutative diagram

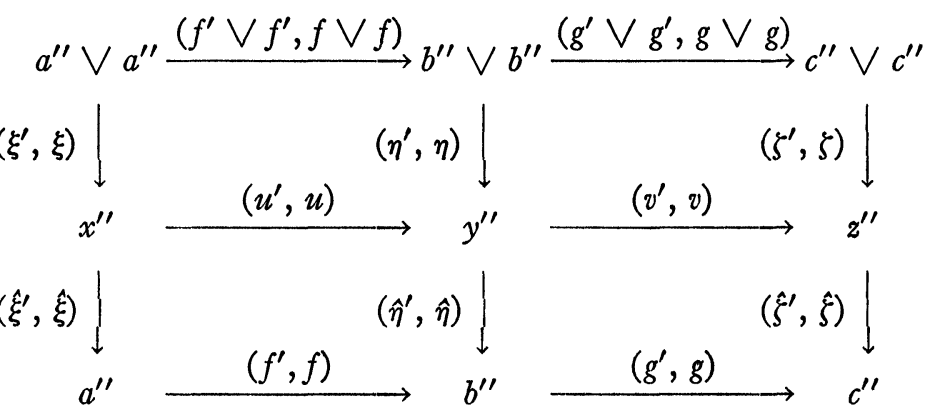

such that

(i) the first two columns are cylinders, with their projections,

(ii) each row is a cofibration, with its cofibre, in Cof $\mathfrak{e}$,

(iii) the first pair of rows is a cofibration, with its cofibre, in $\mathrm{Cof}^{2} \mathfrak{C}$.

It follows, moreover, that the last column is also a cylinder with its projection.

We begin by constructing the first column (Corollary 19.2) and the pushout diagram

$$
\begin{aligned}
& a^{\prime \prime} \vee a^{\prime \prime} \stackrel{\left(f^{\prime} \vee f^{\prime}, f \vee f\right)}{\longrightarrow} \quad b^{\prime \prime} \vee b^{\prime \prime} \\
& \left(\xi^{\prime}, \xi\right) \downarrow \quad \downarrow\left(\tau^{\prime}, \tau\right) \\
& x^{\prime \prime} \longrightarrow\left(t^{\prime}, t\right) \longrightarrow x^{\prime \prime} \cup\left(b^{\prime \prime} \vee b^{\prime \prime}\right) \text {. }
\end{aligned}
$$


Next we apply Lemma 19.1 to

$$
\left(\left(f^{\prime} \hat{\xi}^{\prime}, f \hat{\xi}\right)(11)\right): \quad x^{\prime \prime} \cup\left(b^{\prime \prime} \vee b^{\prime \prime}\right) \rightarrow b^{\prime \prime}
$$

and get a factorization

$$
x^{\prime \prime} \cup\left(b^{\prime \prime} \vee b^{\prime \prime}\right) \stackrel{\left(\theta^{\prime}, \theta\right)}{\longrightarrow} y^{\prime \prime} \stackrel{\left(\hat{\eta}^{\prime}, \hat{\eta}\right)}{\longrightarrow} b^{\prime \prime} .
$$

Notice that $\left(t^{\prime}, t\right)$, as a pushout, is a cofibration. Set $\left(g^{\prime}, g\right)=\left(\theta^{\prime} t^{\prime}, \theta t\right)$, $\left(\eta^{\prime}, \eta\right)=\left(\theta^{\prime} \tau^{\prime}, \theta \tau\right)$. The rest of the diagram is obtained by taking the appropriate cokernels. In particular, as a cofibre in $\operatorname{Cof}^{2} \mathfrak{C},\left(\zeta^{\prime}, \zeta\right)$ is a cofibration in Cof $\mathcal{C}$. The final statement follows by applying Lemma 20.2 to the diagrams obtained from (20.4) by taking domains and ranges of the $a^{\prime \prime}, x^{\prime \prime}$ etc.

Lemma 20.5. If $\left(f^{\prime}, f\right): a^{\prime \prime} \rightarrow b^{\prime \prime}$ in Cof $\mathfrak{e}$ and both $f^{\prime}$ and $f$ are homotopy equivalences in $\mathfrak{C}$, then $\left(f^{\prime}, f\right)$ is a homotopy equivalence in Cof $\mathfrak{C}$.

We consider first the special case in which $\left(f^{\prime}, f\right)$ is a cofibration in Cof $\mathcal{C}$, so that we may construct the diagram (20.4). Under our hypotheses all the morphisms $f^{\prime} \bigvee f^{\prime}, f \bigvee f, u^{\prime}, u, t^{\prime}, t, \theta^{\prime}, \theta$ are homotopy equivalences. By Lemma 19.3, $\left(f^{\prime}, f\right)$ has a retraction $\left(r^{\prime}, r\right)$ and $\left(\theta^{\prime}, \theta\right)$ has a retraction $\left(\rho^{\prime}, \rho\right)$. We define $\left(w^{\prime}, w\right): y^{\prime \prime} \rightarrow b^{\prime \prime}$ as the composition

$$
y^{\prime \prime} \stackrel{\left(\rho^{\prime}, \rho\right)}{\longrightarrow} x^{\prime \prime} \cup\left(b^{\prime \prime} \vee b^{\prime \prime}\right) \stackrel{\left(\left(f^{\prime} \hat{\xi}^{\prime}, f \hat{\xi}\right)\left((1,1)\left(f^{\prime} r^{\prime}, f r\right)\right)\right)}{\longrightarrow} b^{\prime \prime} .
$$

Then $\left(w^{\prime}, w\right)\left(\eta^{\prime}, \eta\right)=\left((1,1)\left(f^{\prime} r^{\prime}, f r\right)\right)$ so that $(1,1) \simeq_{1}\left(f^{\prime} r^{\prime}, f r\right)$.

In general, taking a cylinder $\left(\xi^{\prime}, \xi\right)=\left(\left(\begin{array}{ll}\xi_{0}^{\prime} & \xi_{1}^{\prime}\end{array}\right),\left(\begin{array}{l}\xi_{0} \\ \xi_{1}\end{array}\right)\right): a^{\prime \prime} \bigvee a^{\prime \prime} \rightarrow x^{\prime \prime}$, we consider the diagram

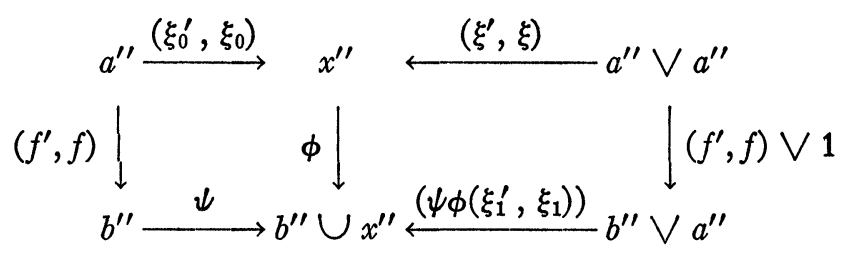

in which the left-hand square is a pushout. By the special case just considered $\psi$ is a homotopy equivalence. But the right-hand square is also a pushout. Thus $\phi\left(\xi_{1}^{\prime}, \xi_{1}\right)$, which is the composition $a^{\prime \prime} \rightarrow b^{\prime \prime} \bigvee a^{\prime \prime}$ $\rightarrow b^{\prime \prime} \cup x^{\prime \prime}$, is a cofibration and thus a homotopy equivalence. Since $\phi\left(\xi_{1}^{\prime}, \xi_{1}\right) \simeq_{1} \phi\left(\xi_{0}^{\prime}, \xi_{0}\right)$, this completes the proof.

We may now proceed to the proof of the theorem. We must show that (Cof $\mathcal{e}, \operatorname{Cof}^{2} \mathcal{C}, \simeq_{1}$ ) satisfies (HC 1-4). The additivity axiom (HC 1) follows immediately from the fact that if $a^{\prime \prime} \vee a^{\prime \prime} \rightarrow x^{\prime \prime}$ and 
$b^{\prime \prime} \cup b^{\prime \prime} \rightarrow y^{\prime \prime}$ are cylinders, then so is $\left(a^{\prime \prime} \bigvee b^{\prime \prime}\right) \bigvee\left(a^{\prime \prime} \bigvee b^{\prime \prime}\right) \rightarrow x^{\prime \prime} \bigvee y^{\prime \prime}$. Axiom (HC 3), deformation-retraction, is an immediate consequence of Lemma 20.5. The mapping cylinder axiom, $\mathrm{HC} 4$, follows at once from Lemmas 19.1 and 20.5.

There remains only the homotopy extension axiom. Suppose

$$
\begin{aligned}
& a^{\prime \prime} \stackrel{\left(f^{\prime}, f\right)}{\longrightarrow} b^{\prime \prime} \stackrel{\left(g^{\prime}, g\right)}{\longrightarrow} c^{\prime \prime} \\
&\left(u^{\prime}, u\right)\left|\left(v^{\prime}, v\right)\right| \mid\left(w^{\prime}, w\right) \\
& \bar{a}^{\prime \prime} \underset{\left(\bar{f}^{\prime}, \vec{f}\right)}{\longrightarrow} b^{\prime \prime} \underset{\left(\bar{g}^{\prime}, \vec{g}\right)}{\longrightarrow} \bar{c}^{\prime \prime}
\end{aligned}
$$

commutes, the rows being cofibrations in Cof $\mathcal{e}$, and that $\left(u_{1}^{\prime}, u_{1}\right)$ $\simeq_{1}\left(u^{\prime}, u\right)$. We may construct the diagram (20.4) and the pushouts

$$
\begin{aligned}
& a^{\prime \prime} \stackrel{\left(f^{\prime}, f\right)}{\longrightarrow} b^{\prime \prime} \quad a^{\prime \prime} \stackrel{\left(f^{\prime}, f\right)}{\longrightarrow} b^{\prime \prime}
\end{aligned}
$$

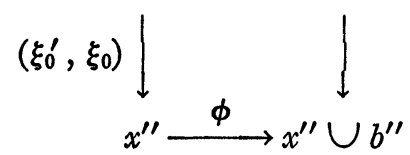

$$
\begin{aligned}
& \begin{aligned}
& \phi\left(\xi_{1}^{\prime}, \xi_{1}\right) \mid \\
& x^{\prime \prime} \cup b^{\prime \prime} \longrightarrow x^{\prime \prime} \cup\left(b^{\prime \prime} \vee b^{\prime \prime}\right) .
\end{aligned}
\end{aligned}
$$

We see that $b^{\prime \prime} \rightarrow x^{\prime \prime} \cup b^{\prime \prime}$ is a homotopy equivalence and that the composition $x^{\prime \prime} \cup b^{\prime \prime} \rightarrow x^{\prime \prime} \cup\left(b^{\prime \prime} \bigvee b^{\prime \prime}\right) \stackrel{\left(\theta^{\prime}, \theta\right)}{\rightarrow} y^{\prime \prime}$ is both a cofibration and a homotopy equivalence, its composition with $b^{\prime \prime} \rightarrow x^{\prime \prime} \cup b^{\prime \prime}$ being $\left(\eta_{0}^{\prime}, \eta_{0}\right)$.

Now since $\left(u_{1}^{\prime}, u_{1}\right) \simeq_{1}\left(u^{\prime}, u\right)$, there is an $\left(\alpha^{\prime}, \alpha\right): x^{\prime \prime} \rightarrow \bar{a}^{\prime \prime}$ giving this homotopy. This defines $\left.\left(f^{\prime} \alpha^{\prime}, f \alpha\right)\left(v^{\prime}, v\right)\right): x^{\prime \prime} \cup b^{\prime \prime} \rightarrow b^{\prime \prime}$ which by the previous remark extends to $\left(\beta^{\prime}, \beta\right): y^{\prime \prime} \rightarrow b^{\prime \prime}$ and gives a commutative diagram

$$
\begin{aligned}
& x^{\prime \prime} \longrightarrow y^{\prime \prime} \longrightarrow z^{\prime \prime} \\
& \left(\alpha^{\prime}, \alpha\right) \downarrow\left(\beta^{\prime}, \beta\right) \downarrow \quad \downarrow\left(\gamma^{\prime}, \gamma\right) \\
& \bar{a}^{\prime \prime} \longrightarrow \bar{b}^{\prime \prime} \longrightarrow \bar{c}^{\prime \prime} .
\end{aligned}
$$

We set $\left(v_{1}^{\prime}, v_{1}\right)=\left(\beta^{\prime}, \beta\right)\left(\eta_{1}^{\prime}, \eta_{1}\right)$ and $\left(w_{1}^{\prime}, w_{1}\right)=\left(\gamma^{\prime}, \gamma\right)\left(\xi_{1}^{\prime}, \xi_{1}\right)$; our conclusion then follows from Lemma 20.3.

We may of course use this result to iterate the construction. If $\left(\mathcal{e}\right.$, Cof $\mathcal{e}, \simeq$ ) is an h-c-category we define $\simeq_{0}=\simeq, \simeq_{n+1}=\left(\simeq_{n}\right)_{1}$ and get the following corollary to Theorem 20.1.

Corollary 20.6. If (e, Cof $\mathfrak{e}, \simeq$ ) is an h-c-category, then so also is $\left(\operatorname{Cof}^{n} \mathcal{e}, \operatorname{Cof}^{n+1} \mathcal{C}, \simeq_{n}\right)$ for $n=0,1,2, \cdots$. 


\section{REFERENCES}

1. J. F, Adams, Stable homotopy theory, Lecture Notes in Math., no. 3, SpringerVerlag, New York, 1966.

2. J. M. Boardman, Stable homotopy theory, University of Warwick, 1966.

3. E. H. Brown, Cohomology theories, Ann. of Math. (2) 75 (1962), 467-484.

4. - - Abstract homotopy theory, Conference on Algebraic Topology, Seattle, 1963.

5. A. Dold, Relations between ordinary and extraordinary homology, Colloquium on Algebraic Topology, Matematisk Institut, Aarhus Universitat, Aarhus, 1962, pp. 1-9.

6. - Halbexakte Homotopiefunktoren, Lecture Notes in Math., no. 12, Springer-Verlag, New York, 1966.

7. S. Eilenberg and J. C. Moore, Foundations of relative homological algebra, Mem. Amer. Math. Soc. No. 55 (1965).

8. S. Eilenberg and G. M. Kelly, Closed categories, Proc. Conf. on Categorical Algebra, La Jolla 1965, Springer-Verlag, New York, 1966, pp. 421-562.

9. P. J. Freyd, Stable homotopy, Proc. Conf. on Categorical Algebra, La Jolla 1965, Springer-Verlag, New York, 1966, pp. 121-172.

10. - - Splitting homotopy idempotents, ibid., pp. 173-176.

11. - The Grothendieck group for stable homotopy is free, Bull. Amer. Math. Soc. 73 (1967), 84-86.

12. A. Heller, Homological algebra in abelian categories, Ann. of Math. (2) 68 (1958), 484-525.

13. —- The loop-space functor in homological algebra, Trans. Amer. Math. Soc. 96 (1960), 382-394.

14. - Extraordinary homology and chain complexes, Proc. Conf. on Categorical Algebra, La Jolla 1965, Springer-Verlag, New York, 1966, pp. 355-365.

15. D. M. Kan, Abstract homotopy. II, Proc. Nat. Acad. Sci. U.S.A. 42 (1956), 255-258.

16. D. Puppe, Homotopiemengen und ihre induzierte Abbildungen. I, Math. Z. 69 (1958), 299-344.

17. - Stabile Homotopietheorie. I, Math. Ann. 169 (1967), 243-274.

18. E. Spanier, Secondary operations on mappings and cohomology, Ann. of Math. (2) 75 (1962), 260-282.

19. E. H. Spanier and J. H. C. Whitehead, Duality in homotopy theory. Mathematika 2 (1955), 58-60.

20. H. Toda, Composition methods in homotopy groups of spheres, Ann. of Math. Studies, no. 49, Princeton Univ. Press, Princeton, N. J., 1962.

21. J. Verdier, Catégories derivées, quelques resultats, Mimeographed Notes, I.H.E.S., Paris, 1966.

22. G. W. Whitehead, Generalized homology theories, Trans. Amer. Math. Soc. 102 (1962), 227-283. 